\title{
Nano intervention in topical delivery of corticosteroid for psoriasis and atopic dermatitis - a systematic review
}

\author{
Kshitya Shetty ${ }^{1}$ Atul P. Sherje $\mathbb{A D}^{1}$ \\ Received: 9 September 2020 / Accepted: 7 July 2021 / Published online: 31 July 2021 \\ (c) The Author(s) 2021
}

\begin{abstract}
Atopic dermatitis (AD) and psoriasis are highly prevalent, complex, chronic inflammatory skin diseases that immensly affect the patient's quality of life. While there is no definitive cure for these conditions, suppressive medications aim at managing the symptoms of these diseases. The application of emollients accompanied by symptomatic anti-inflammatory therapy consisting of topical corticosteroids (TCS) is extensively employed for controlling the symptoms among general practitioners making this therapeutic class an indispensable pillar of dermatotherapeutics. The first TCS, hydrocortisone (HC) introduced in the early 1950s led to the development of different steroidal moieties of varying potencies by inducing chemical modifications to the basic steroid structure. The wide spectrum of the available range of formulations and potency provides flexibility to treat all patient groups, different phases of the diseases, and different anatomical sites. Conventional TCS therapy suffers from drawbacks such as low drug permeation and retention rate. Thus, novel nanoformulations have been developed to overcome these problems. This review provides an insight into the current state of nanocarrier-mediated topical delivery of corticosteroids monotherapy and combination therapy with special emphasis on targeting psoriasis and $\mathrm{AD}$.
\end{abstract}

\section{Graphical Abstract}

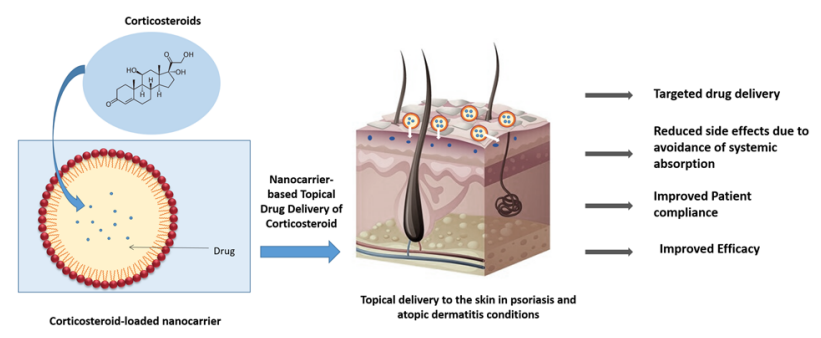

\section{Highlights}

- Psoriasis and atopic dermatitis (AD) are highly prevalent and chronic skin disorders.

- Topical corticosteroids (TCS) is extensively utilized as a drug of choice for such diseases.

- Extensive research has been conducted to formulate and evaluate a nanocarrier-based drug delivery system.

- These TCS nanoformulations in combination therapy involving other therapeutic classes of drugs is also been extensively researched for effective disease management.

- This manuscript gives an insight into the nanotechnology-based topical formulations of corticosteroids intended for AD and psoriasis therapy.

Atul P. Sherje

sherjeap@gmail.com

1 SVKM's Dr. Bhanuben Nanavati College of Pharmacy, Mumbai 400 056, India

$\begin{array}{ll}\text { Abbreviations } \\ \text { AD } & \text { atopic dermatitis } \\ \text { TCS } & \text { topical corticosteroids } \\ \text { FDA } & \text { Food and Drug Administration } \\ \text { SC } & \text { stratum corneum } \\ \text { TEWL } & \text { transepidermal water loss } \\ \text { FLG } & \text { filaggrin }\end{array}$




\begin{tabular}{|c|c|c|c|}
\hline $\mathrm{TJ}$ & tight junctions & NTPDase & nucleoside triphosphate diphosphohydroalse \\
\hline CLDN1 & Claudin-1 & MF & mometasone furoate \\
\hline IL & interleukin & LNC & lipid-core nanocapsules \\
\hline $\operatorname{IgE}$ & immunoglobulin $\mathrm{E}$ & $\mathrm{CMC}$ & critical micelle concentration \\
\hline \multirow[t]{2}{*}{ NFkB } & nuclear factor kappa-light-chain-enhancer of & DFV & diflucortolone valerate \\
\hline & activated B cells & BMV & betamethasone-17-valerate \\
\hline $\mathrm{C} / \mathrm{EBP}$ & CCAAT/enhancer-binding protein & BDP & betamethasone dipropionate \\
\hline STAT & Signal transducer and activator of transcription & $\mathrm{CP}$ & clobetasol propionate \\
\hline HSP & heat-shock proteins & BDP-Ch- & betamethasone dipropionate/sodium cholate \\
\hline GR & glucocorticoid receptor & LDH & intercalated layered double hydroxide \\
\hline PEPCK & phosphoenolpyruvate carboxykinase & QbD & quality by design \\
\hline TAT & tyrosine aminotransferase & HPMC & hydroxypropyl methylcellulose \\
\hline DUSP-1 & dual specificity protein phosphatase 1 & $\mathrm{PC}$ & soybean phosphatidylcholine \\
\hline \multirow[t]{2}{*}{$\mathrm{NF}-\kappa \mathrm{B}$} & nuclear factor kappa-light-chain-enhancer of & DMPC & dimyristoylphosphatidylcholine \\
\hline & activated $B$ cells & FTIR & Fourier-transform infrared spectroscopy \\
\hline GRE & glucocorticoid response elements & $\mathrm{LC}$ & lecithin/chitosan nanoparticles \\
\hline ANXA1 & annexin A1 & $\mathrm{SBE} \beta \mathrm{CD}$ & sulfobutylether- $\beta$-cyclodextrin \\
\hline PLA2 & phospholipase A2 & $\mathrm{CS}$ & chitosan \\
\hline $5 \mathrm{HT}$ & 5-hydroxytryptamine & NPs & nanoparticles \\
\hline \multirow[t]{2}{*}{ GM-CSF } & granulocyte-macrophage colony- & PNIPAAm-- & poly (N-isopropylacrylamide-dl-lactide) \\
\hline & stimulating factor & PLA & copolymer block \\
\hline SLN & solid lipid nanoparticles & LCST & lower critical solution temperature \\
\hline NLC & nanostructured lipid carriers & PLGA & poly (d,1-lactic/glycolic acid) \\
\hline $\mathrm{Tg}$ & transition temperature & PLA & $\operatorname{poly}(\mathrm{d}, 1-$ lactic acid $)$ \\
\hline $\mathrm{CD}$ & cyclodextrin & PCL & poly ( $\varepsilon$-caprolactone) \\
\hline $\mathrm{HP} \gamma \mathrm{CD}$ & 2-hydroxypropyl- $\gamma$-cyclodextrin & TEM & transmission electron microscopy \\
\hline $\mathrm{PC}$ & phosphatidylcholine & SBA-15 & hexagonal mesoporous silica nanoparticles \\
\hline DMPC & dimyristoylphosphatidylcholine & APTES & (3-aminopropyl) triethoxysilane \\
\hline LDH & layered double hydroxide & PDI & polydispersity index \\
\hline $\mathrm{Ch}$ & sodium cholate & $\mathrm{HC}$ & hydrocortisone \\
\hline LS & liposome & HT & hydroxytyrosol \\
\hline BMV & betamethasone valerate & $\mathrm{HC}-\mathrm{HT}$ & hydrocortisone and hydroxytyrosol \\
\hline DFV & diflucortolone valerate & $\mathrm{CS}$ & chitosan \\
\hline TEWL & transepidermal water loss & TPP & pentasodium tripolyphosphate ions. \\
\hline SEM & scanning electron microscopy & LD 50 & median lethal dose \\
\hline DSC & differential scanning calorimetry & $\mathrm{HaCaT}$ & cultured human keratinocyte cells \\
\hline TA & triamcinolone acetonide & PDCs & peptide-drug conjugates \\
\hline
\end{tabular}

DOTAP 1,2-dioleoyl-3-trimethylammonium-propane

DOPE dioleoylphosphatidyl ethanolamine

FA fluocinolone acetonide

GMS glycerol monostearate

FP fluticasone propionate

HP halobetasol propionate

HP-NLC halobetasol propionate-loaded NLC

$\mathrm{CP} \quad$ clobetasol propionate

CP-NLC clobetasol propionate-loaded nanostructured lipid carriers

CP-NLC-C chitosan-coated clobetasol propionate-loaded nanostructured lipid carriers

$\mathrm{O} / \mathrm{W} \quad$ oil in water

W/O water in oil

BD betamethasone dipropionate

PEG polyethylene glycol

\section{Introduction}

Topical corticosteroids (TCS) are a class of FDA-approved prescription drugs that play a vital role in the treatment of inflammatory and pruritic presentations of dermatologic conditions such as psoriasis, dermatitis, eczema, limited areas of vitiligo, and lichen sclerosis [1,2]. Their clinical effectiveness in the treatment of these dermatological disorders is related to their vasoconstrictive, anti-inflammatory, immunosuppressive, and antiproliferative effects. The desired therapeutic responses of a drug with minimum adverse effects by administration via topical or transdermal delivery are achieved by overcoming the formidable stratum corneum (SC) barrier. The SC has an array of flattened 
corneocyte layers enclosed by a lipid envelope, which assists the skin to perform its permeability barrier function [3]. The drug molecules permeate through this layer mainly via the paracellular, transcellular, and transappendgeal routes. The substances suited for diffusion through SC are small (Mol. Wt. $\leq 400 \mathrm{Da}$ ), lipophilic drug ( $\log k>3$; favorable via intracellular route), and hydrophilic drugs ( $\log k<1$; favorably permeated via transcellular route) [4]. The major drawbacks of topical drug delivery are the potential of skin irritation, allergic reactions, degradation of drugs by the enzymes present in the epidermis, or other topical side effects arising from its prolonged drug use in the topical formulation [5]. Traditionally, conventional therapy options such as creams, ointments, lotions, etc. show limited accessibility to the deeper layers of the skin [6]. Therefore, to overcome all these shortcomings, a nanotechnology-based drug delivery system that carries a charge to increase flux through the skin or lipid coating to boost retention in the SC has been demonstrated to amplify the bioavailability of lipophilic drugs [7]. The most extensively used nano-drug delivery systems include lipid-based nanoparticles (NPs) i.e., liposomes, noisome, ethosomes, nanoemulsions, solid lipid nanoparticles (SLN), nanostructured lipid carriers (NLC), lipid nanocapsules, or polymer-based nanocarriers like (polymeric NPs, polymeric micelles, polymer-drug conjugates) and peptide-drug conjugates (PDCs) [5]. Thus, this paper is a comprehensive review of the recent developments in various nano-drug delivery systems for topical delivery of corticosteroid with special emphasis on $\mathrm{AD}$ and psoriasis skin conditions as an effective palliative therapy [7].

\section{Pathophysiology}

TCS is often the drug of choice for two major diseases namely $\mathrm{AD}$ and psoriasis. These dermal conditions are inflammatory leading to lesions, rashes, declined skin barrier action, and psychosocial issues [8].

\subsection{Pathophysiology of AD}

Erythemic skin rashes, infected with staphylococcus aureus bacteria, are the characteristic symptoms of $\mathrm{AD}$ [9]. $\mathrm{AD}$ is prevalent during childhood affecting up to $20 \%$ of children and up to $3 \%$ of adults in developed countries and the number is estimated to be higher in the case of low-income nations [10]. The exact etiology of AD is unknown, but they are both genetic and environmental factors involved in the pathogenesis of the disease. Skin barrier dysfunction in $\mathrm{AD}$ occurs due to decreased levels of total ceramides and changes in its composition (changes in ceramide chain length, free fatty acids, and esterified fatty acids), which disrupts epidermal lipid organization; decreased expression of other epidermal differentiation-related molecules such as filaggrin (FLG), loricrin, involucrin production. Decreased production of FLG is attributed to a high rate of FLG mutation (up to $47 \%$ ) as observed among the European patient population. FLG abnormalities cause increased transepidermal water loss (TEWL), which affects SC hydration [11-13]. The tight junctions (TJ) of keratinocytes in the epidermal granule layer, act as a second physical barrier in the epidermis. TJ are adhered together by Claudin-1 (CLDN1) adhesion protein, hence deficiencies in CLDN1 expression in the TJ of the upper epidermis and the presence of single nucleotide polymorphisms in the CLDN1 gene are commonly observed among patients with $\mathrm{AD}$ [14]. Another indication of $\mathrm{AD}$ is the elevated helper T-cell type-2 cytokines (interleukin (IL)4, IL-5, IL-13, IL- 22, IL-25, and IL-33) levels, causes an increase in serum immunoglobulin E (IgE) level and also linked to the chronicity and amplification of skin inflammation in $\mathrm{AD}$ [13]. This deterioration of skin barrier action increases susceptibility to environmental triggers and staphylococcus aureus infection, which are linked to $\mathrm{AD}$. Current therapies aim at reducing inflammation, rebuilding the skin barrier, and fighting bacterial infection [9].

\subsection{Pathophysiology of psoriasis}

Psoriasis is an autoimmune disorder that leads to the formation of plaques, erythemic skin rashes, scaling, and hyperproliferation of keratinocytes [15]. Statistical records suggest that psoriasis has a chance of development at any age and its occurrence depends on genetic and environmental triggers. It affects $2-5 \%$ of the adult population globally, in the form of plaque or guttate psoriasis [16]. The various dermatological manifestations of psoriasis are classified as follows: Psoriasis Vulgaris being the most prevalent (90\%), which corresponds to the chronic plaque-type psoriasis with sharply demarcated, erythematous, silvery-scaled pruritic plaques covering large areas of the skin of the trunk, limbs, and scalp.

Inverse psoriasis, also known as flexural psoriasis, affects the intertriginous location and is characterized by slightly erosive erythematous plaques and patches. Guttate psoriasis is an acute onset of small erythematous plaques and usually affects children and adolescents. Pustular psoriasis is characterized by localized or generalized multiple, coalescing sterile pustules. Types of pustular psoriasis include pustulosa palmoplantaris which occurs in palms and soles and acrodermatitis continua of Hallopeau, which is more distally located at the tips of fingers, nails, and toes. Last, erythrodermic psoriasis develops in any kind of psoriasis and requires emergency treatment; it is an acute condition in which over $90 \%$ of the total body surface is erythematous and inflamed [17]. The exact etiology of psoriasis is unclear but it is associated with mutations to the late cornified envelope genes or the major histocompatibility complex. The presence 
of genetic factors (human leukocyte antigen Cw6 gene; psoriasis susceptibility 1 gene) and/or environmental triggers like stress, smoking, alcohol intake, infections (60\% guttate psoriatic patient have experienced preceding upper respiratory tract infections), presence of diseases like HIV, administration of certain drugs (notably lithium, $\beta$-adrenoceptor blockers, antimalarial agents related to chloroquine) could contribute in inducing psoriatic damage to the skin barrier [18]. Thus, in response to the repair mechanism, the skin keratinocyte hyperproliferate without proper differentiation resulting in the formation of poor skin barrier and development of scaly plaques. Inflammation in psoriasis is intervened by $\mathrm{T}$ helper cells (Type-17, Type-22, and Type-1) causing increased levels of cytokine in the skin (interferon-g, IL-12, IL-17, IL-22). Cytokines synthesized by activated Type-17 $\mathrm{T}$ helper cells include IL-17 (IL-17A/IL-17F), IL-26, IL-29, and TNF, which activate nuclear factor kappa-light-chain-enhancer of activated $B$ cells $(\mathrm{NFkB}), \mathrm{CCAAT} / \mathrm{enhancer}^{-b i n d i n g}$ protein $(\mathrm{C} / \mathrm{EBP} \beta)$ or (C/EBPS), and signal transducer and activator of transcription 1 in keratinocytes causing an inflammatory response and further activation and recruitment of $\mathrm{T}$ helper cells (Type22 and Type-1) subsets into psoriatic lesions. Epidermal hyperplasia in psoriasis is associated with STAT3 activation, is induced by IL-17 through increased production of IL-19 and IL-36 in epidermal keratinocytes [19-22].

Based on pathophysiological mechanism, psoriasis is distinguished from $\mathrm{AD}$ by (a) the absence of $\mathrm{T}$ helper cells (Type-2) that synthesize IL-4, IL-5, and IL-13, (b) the absence of IgE antibodies, which are strongly dependent on activated T helper cells (Type-2). Activated B cells are largely absent in psoriasis patients, whereas activated $\mathrm{B}$ cells that produce $\mathrm{IgE}$ are commonly seen in the circulation of $\mathrm{AD}$ patients, (c) presence of comorbidities in psoriatic patients such as psoriatic arthritis, obesity, metabolic dysregulation, and cardiovascular disease that stem from inflammatory etiologies [23].

Current treatment options for both psoriasis and $\mathrm{AD}$ primarily focus on reducing the inflammation and repair of the skin barrier. Mild-to-moderate psoriasis can be treated topically with glucocorticoids, vitamin D analogs, phototherapy, and a combination of the same. Whereas moderate-to-severe psoriasis often requires systemic therapies such as smallmolecule-(methotrexate, cyclosporin A, and retinoids), biologic drugs (etanercept, adalimumab, certolizumab, infliximab, brodalumab, ustekinumab, guselkumab ixekizumab, secukinumab, etc.), and their biosimilars (adalimumab biosimilars, four infliximab biosimilars, and two etanercept biosimilars) [24].

\section{TCS for AD and psoriasis}

Both the diseases mentioned above are incurable and non-life threatening. However, they are faced with expensive treatment options and cause psychosocial stigma to the patients. Since both these conditions are characterized by similar symptoms, the line of therapy overlaps significantly. TCS is efficient in alleviating the symptoms, but nanocarrierbased formulation could further advance the therapy by enhancing drug permeation, skin retention, thereby reducing the dosing frequency, lowering drug concentration, and abating adverse events $[8,25,26]$.

\subsection{Mechanism of action}

The adrenal cortex is one of the major endocrine glands present in the body and its secretion includes glucocorticoids, mineralocorticoids, and sex hormones. Corticosteroids are steroid hormones that are either derived from the adrenal cortex or synthetically obtained from it. TCS mainly comprises glucocorticoids, which possess immunomodulatory, apoptotic, anti-inflammatory, gluconeogenic, vasoconstrictor, and antimitotic activity. These actions are mediated at a cellular level through genomic and nongenomic mechanisms [27, 28].

\subsubsection{Genomic pathway}

The genomic pathway involves activation of the glucocorticoid receptor (GR). The GR is found in most of the cells of the body, which accounts for the widespread systemic effects of TCS. In the skin, GR is located in keratinocytes and fibroblasts within the epidermis and dermis. When the receptors are unoccupied by the corticosteroid molecule, they are usually present in the cell cytoplasm. The inactive receptor is bound to proteins like heat-shock proteins (HSP) like HSP 90, HSP 70, immunophilins, cyclophilins, and calreticulin protein. The lipophilic glucocorticoid molecule enters the cell via passive diffusion. Within the cell, it binds to the receptor, by dissociation of the inactive receptor and HSP, immunophilins complex and the corticosteroid-receptor complex then translocates to the nucleus wherein it undergoes dimerization. This dimer complex further binds to glucocorticoid response element (i.e., a specific palindromic promoter DNA sequence) and helps induce transcription of genes with anti-inflammatory functions such as promotion of phosphoenolpyruvate carboxykinase, $\beta$-adrenergic receptor, tyrosine aminotransferase, IL-1-receptor antagonist, IL-10, and dual-specificity protein phosphatase-1 [29].

\subsubsection{Non-genomic pathway}

The non-genomic pathway involves direct interaction of liganded GR with diverse intracellular mediators and modulating several signaling pathways, including protein kinase $\mathrm{C}$, phosphatidylinositol-specific phospholipase C. It also involves 


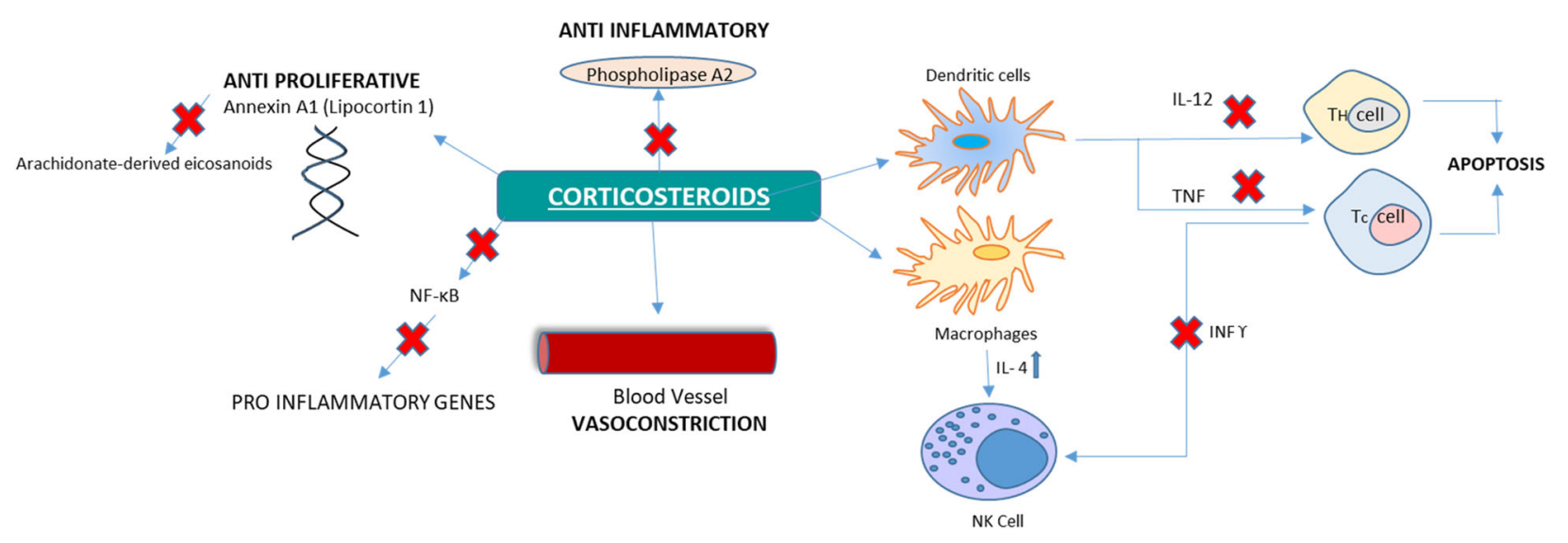

Fig. 1 Mechanism of action of TCS

the management of activation and responsiveness levels of target cells, such as monocytes, T cells, and platelets. This pathway is responsible for the rapid effects of glucocorticoids that occur within a few minutes [29].

\subsection{Therapeutic properties of TCS}

Corticosteroids are useful in varied dermatological conditions due to their anti-inflammatory, immunosuppressant, vasoconstrictive, and antiproliferative effect.

The anti-inflammatory activity of TCS occurs due to the expression of annexin A1 protein binds to phospholipids, thereby reducing inflammatory prostanoid production. It also causes

(a) inhibition of phospholipase A2 thus obstructing the synthesis of arachidonate-derived eicosanoids (prostaglandins, prostacyclins, leukotrienes, and thromboxanes),

(b) inhibition of cyclooxygenase induction resulting in decreased prostaglandin production,

(c) inhibition of nitric oxide synthase induction, which decreases nitric oxide production,

(d) inhibition of cytokines production causing suppression of cell-mediated inflammation,

(e) inhibition of mass cell activity and reduction of mast cell, thus decreasing the levels of mast cell inflammatory mediators, and

(f) vasoconstriction decreases local blood flow [27].

Topical glucocorticoids such as dexamethasone are considered to have an antiproliferative effect over the A549 cell line, which is associated with an increase of annexin A1 causing a decrease in the cell turnover rate of the skin in the case of psoriasis. These agents also exhibit a decrease in the survival of eosinophils and lymphocytes. The apoptosis of eosinophils is also associated with blockade of the IL-5 and granulocyte-macrophage colony-stimulating factor effects. Alternatively, glucocorticoids enhance neutrophil survival. Vasoconstriction, also known as blanching, is a part of the anti-inflammatory effects of glucocorticoid. A decline in blood circulation to the inflamed site can be used as a standard assay for the evaluation of the drug potency. The underlying vasoconstrictor mechanism involves blocking vasodilators like histamine and bradykinin [29].

These drugs also inhibit humoral factors involved in the inflammatory response, repress maturation, differentiates and proliferates all immune cells, comprising dendritic cells and macrophages thereby decreasing $\mathrm{T}$ helper 1-cellinducing cytokine IL-12 production, hinder in the function of endothelial cells, granulocytes, and fibroblasts. These drugs inhibit leukocyte migration to sites of inflammation. Furthermore, these steroids have a high chance of amplifying delayed-type hypersensitivity [27]. The mechanism of action of topical glucocorticoids is illustrated in Fig. 1.

\subsection{Classification of TCS}

TCS was first classified in 1985 by Stoughton-Cornell in the decreasing order of their potency and therapeutic index. Subsequently, other modes of drug classification were introduced based on chemical structure i.e., halogenated and nonhalogenated. Examples of nonhalogenated corticosteroids include HC (and its derivatives), desonide, prednicarbate, and methylprednisolone aceponate [30]. The latest WHO classification of TCS divides them into seven classes/groups in decreasing order of their potency. In this system, potency is based on the activity of the TCS molecule, its concentration, and the nature of the vehicle used for drug administration. The same drug can be placed into multiple classes due to varying potencies with the use of different vehicles. In this classification, the seven classes of corticosteroids are further categorized into four subgroups, wherein class I is considered as ultra-high 
potency, classes II and III as high-potency, classes IV and $\mathrm{V}$ as moderate-potency, and classes VI and VII as lowpotency corticosteroids.

The UK-based classification (British National Formulary) has four classes of TCS-Class I being the most potent, while class IV includes drugs with low potency. TCS of lower potency are particularly recommended for less resistant plaque treatment and applied on the groin, face, axillary areas of adults, infants, and children. Mid and higher potency corticosteroids are employed for the use of initial therapy of dermal disorders on other body areas in adults. Chronic or hyperkeratotic $\mathrm{AD}$ lesions and cutaneous psoriatic plaques on the scalp, palms, and soles are treated by the use of super potent corticosteroids for short-term use (maximum period of at least 2 weeks) to minimize the risk of steroidal side effects [29]. The drug classification and their vehicles based on their potency are mentioned in Table 1.

\subsection{Clinical adverse effects of TCS}

TCS is employed in the therapy of many dermatological ailments but they have a potential for adverse effects. The severity and occurrence of these adverse effects are dependent on the specific drug used, duration of action, dosage, dosing regime, and individual patient variability. However, prolonged use of the drug is the highest risk factor involved in causing side effects. Prolonged therapy with TCS also increases systemic drug concentration leading to an occurrence of systemic side effects such as weight gain, hypothalamopituitary-adrenal axis suppression, iatrogenic Cushing's syndrome, etc. and local side effects like bruising, skin irritation, epidermal atrophy, hypopigmentation in the treated area, photosensitivity, premature aging, steroid-induced acne, rosacea [31, 32].

\subsection{Conventional drug delivery systems of TCS}

TCS is formulated into different conventional vehicles like ointments, creams, lotions, gels, solutions, and newer formulations such as shampoos and foams [30]. The summary of various conventional formulations of TCS is depicted in Table 2.

\section{Nanocarrier-mediated delivery of TCS in psoriasis and AD}

Conventional dosage forms are extensively marketed for the therapy of dermatological conditions but they suffer from massive problems such as skin irritation, low drug permeation through scaly skin, prolonged dosage regimens thus rendering it less efficacious for therapy [33]. Currently, nanotechnology-based formulations are gaining immense
Table 1 WHO classification of TCS and commercial formulations [128]

\begin{tabular}{|c|c|c|c|}
\hline Potency & Class & Topical corticosteroid & Formulation \\
\hline \multirow[t]{2}{*}{ Ultra-high } & I & Clobetasol propionate & Cream, $0.05 \%$ \\
\hline & & Diflorasone diacetate & Ointment, $0.05 \%$ \\
\hline \multirow[t]{9}{*}{ High } & II & Amcinonide & Ointment, $0.1 \%$ \\
\hline & & $\begin{array}{l}\text { Betamethasone } \\
\text { dipropionate }\end{array}$ & Ointment, $0.05 \%$ \\
\hline & & Desoximetasone & $\begin{array}{l}\text { Cream or } \\
\text { ointment, } 0.025 \%\end{array}$ \\
\hline & & Fluocinonide & $\begin{array}{l}\text { Cream, ointment, or } \\
\text { gel, } 0.05 \%\end{array}$ \\
\hline & & Halcinonide & Cream, $0.1 \%$ \\
\hline & III & $\begin{array}{l}\text { Betamethasone } \\
\text { dipropionate }\end{array}$ & Cream, $0.05 \%$ \\
\hline & & Betamethasone valerate & Ointment, $0.1 \%$ \\
\hline & & Diflorasone diacetate & Cream, $0.05 \%$ \\
\hline & & Triamcinolone acetonide & Ointment, $0.1 \%$ \\
\hline \multirow[t]{12}{*}{ Moderate } & IV & Desoximetasone & Cream, $0.05 \%$ \\
\hline & & Fluocinolone acetonide & Ointment, $0.025 \%$ \\
\hline & & Fludroxycortide & Ointment, $0.05 \%$ \\
\hline & & Hydrocortisone valerate & Ointment, $0.2 \%$ \\
\hline & & Triamcinolone acetonide & Cream, $0.1 \%$ \\
\hline & $\mathrm{V}$ & $\begin{array}{l}\text { Betamethasone } \\
\text { dipropionate }\end{array}$ & Lotion, $0.02 \%$ \\
\hline & & Betamethasone valerate & Cream, $0.1 \%$ \\
\hline & & Fluocinolone acetonide & Cream, $0.025 \%$ \\
\hline & & Fludroxycortide & Cream, $0.05 \%$ \\
\hline & & Hydrocortisone butyrate & Cream, $0.1 \%$ \\
\hline & & Hydrocortisone valerate & Cream, $0.2 \%$ \\
\hline & & Triamcinolone acetonide & Lotion, $0.1 \%$ \\
\hline \multirow[t]{6}{*}{ Low } & VI & Betamethasone valerate & Lotion, $0.05 \%$ \\
\hline & & Desonide & Cream, $0.05 \%$ \\
\hline & & Fluocinolone acetonide & Solution, $0.01 \%$ \\
\hline & VII & $\begin{array}{l}\text { Dexamethasone sodium } \\
\text { phosphate }\end{array}$ & Cream, $0.1 \%$ \\
\hline & & Hydrocortisone acetate & Cream, $1 \%$ \\
\hline & & $\begin{array}{l}\text { Methylprednisolone } \\
\text { acetate }\end{array}$ & Cream, $0.25 \%$ \\
\hline
\end{tabular}

applicability in the field of dermatology. Due to enhanced surface area, nanocarrier-based drug delivery systems have been suggested as the preferred drug delivery system since they help overcome the issues of conventional dosage form and also provide a safe, targeted, and controlled release dosage form [6, 33]. During the formulation of a nanocarrier-based drug delivery system various parameters like surface charge, size and morphology of NPs, entrapment efficiency, and drug permeation are considered vital to formulate an effective product [6]. Nanoformulations generally vary in size from 10 to $100 \mathrm{~nm}$, the smaller-sized formulation increases the bioavailability and solubility of 


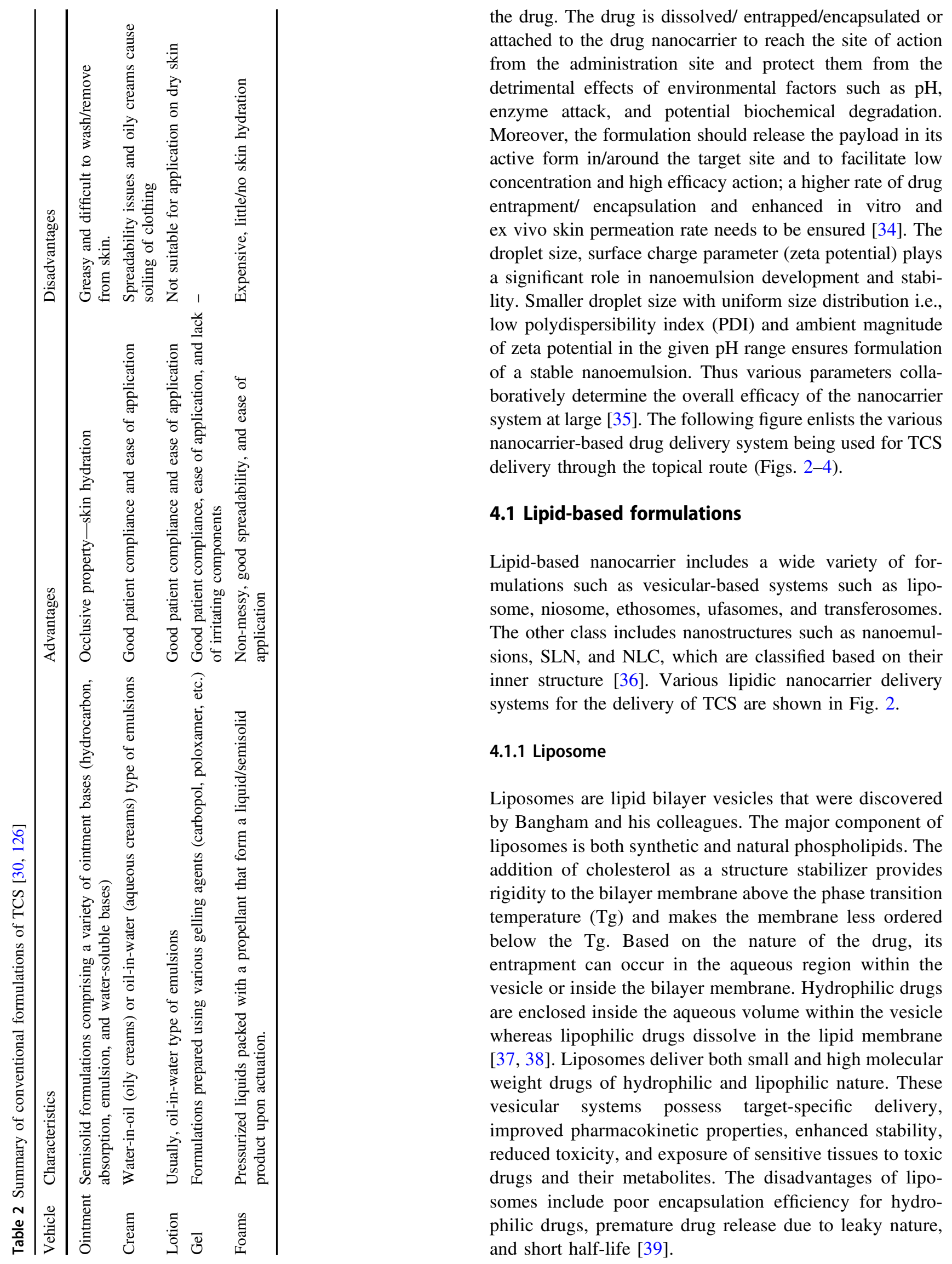

the drug. The drug is dissolved/ entrapped/encapsulated or attached to the drug nanocarrier to reach the site of action from the administration site and protect them from the detrimental effects of environmental factors such as $\mathrm{pH}$, enzyme attack, and potential biochemical degradation. Moreover, the formulation should release the payload in its active form in/around the target site and to facilitate low concentration and high efficacy action; a higher rate of drug entrapment/ encapsulation and enhanced in vitro and ex vivo skin permeation rate needs to be ensured [34]. The droplet size, surface charge parameter (zeta potential) plays a significant role in nanoemulsion development and stability. Smaller droplet size with uniform size distribution i.e., low polydispersibility index (PDI) and ambient magnitude of zeta potential in the given $\mathrm{pH}$ range ensures formulation of a stable nanoemulsion. Thus various parameters collaystem at large [35]. The following figure enlists the various nanocarrier-based drug delivery system being used for TCS mulations such as vesicular-based systems such as liposome, niosome, ethosomes, ufasomes, and transferosomes. systems for the delivery of TCS are shown in Fig. 2.

\subsubsection{Liposome}

Liposomes are lipid bilayer vesicles that were discovered by Bangham and his colleagues. The major component of posomes is both synthetic and natural phospholipids. The provides transition ordered low the Tg. Based on the nature of the drug, its rapment can occur in the aqueous region within the sicle or inside the bilayer membrane. Hydrophilic drugs enclosed inside the aqueous volume within the vesicle hereas lipophilic drugs dissolve in the lipid membrane 37, 38]. Liposomes deliver both small and high molecular weight drugs of hydrophilic and lipophilic nature. These esicular systems possess target-specific delivery, mproved pharmacokinetic properties, enhanced stability, reduced toxicity, and exposure of sensitive tissues to toxic drugs and their metabolites. The disadvantages of liposomes include poor encapsulation efficiency for hydroand short half-life [39] 


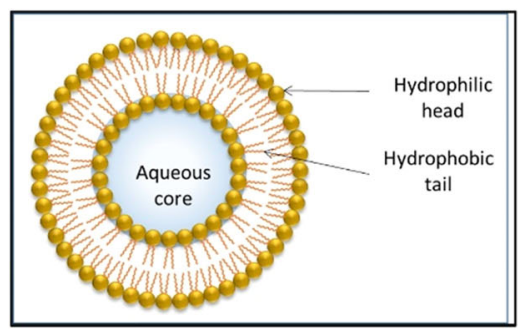

Liposome

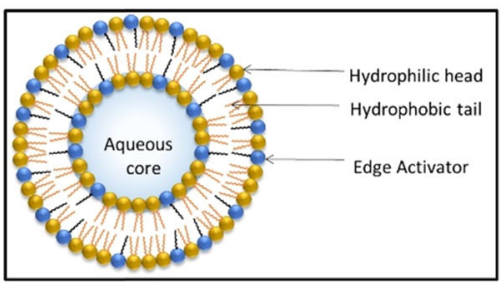

Transferosomes

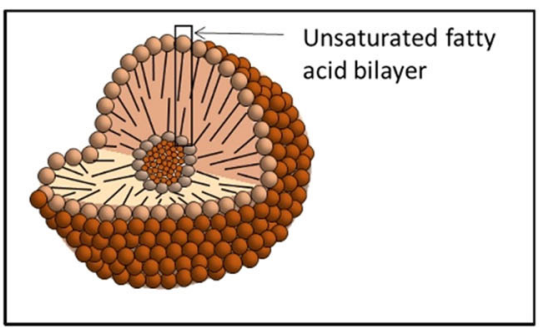

Ufasome

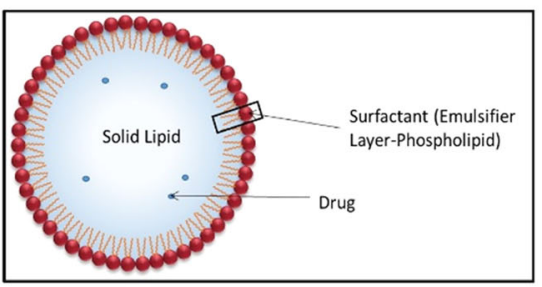

Sold lipid nanoparticles

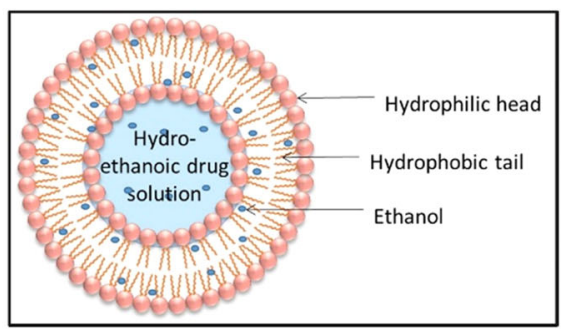

Ethosome

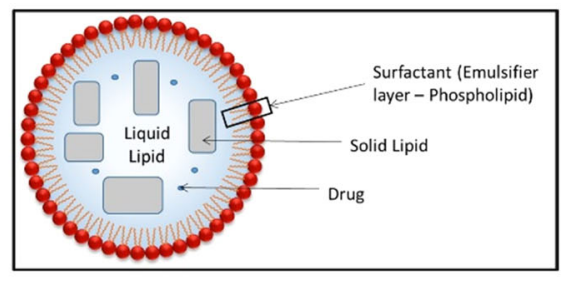

Nanostructured Lipid Carrier

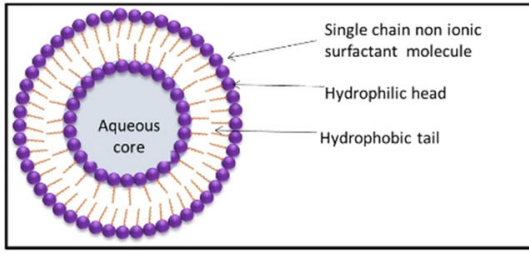

Niosome

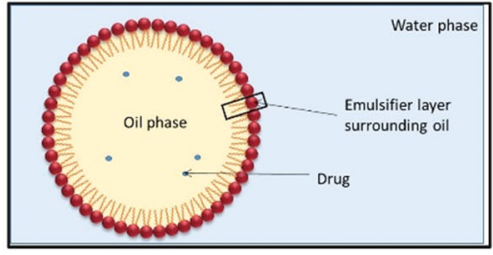

Nanoemulsion

Fig. 2 Lipidic nanocarrier delivery systems for TCS

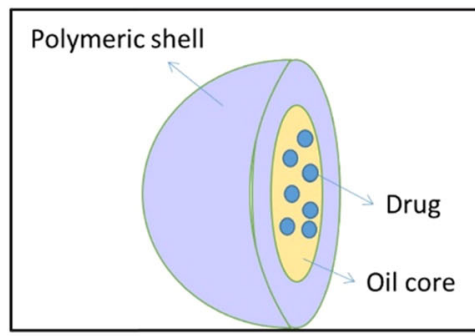

Polymeric capsule

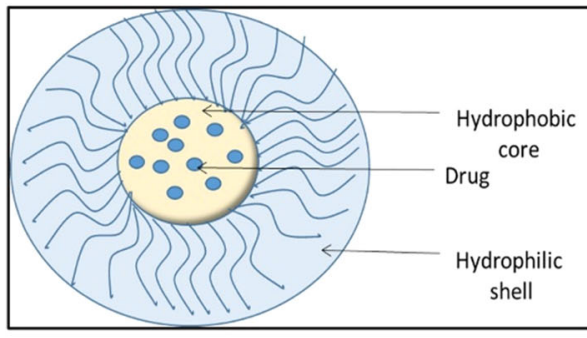

Polymeric micelle

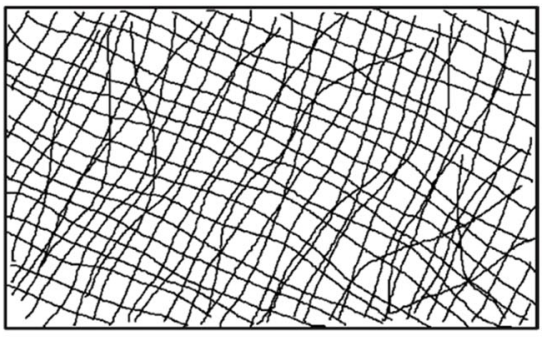

Polymeric nanofibers

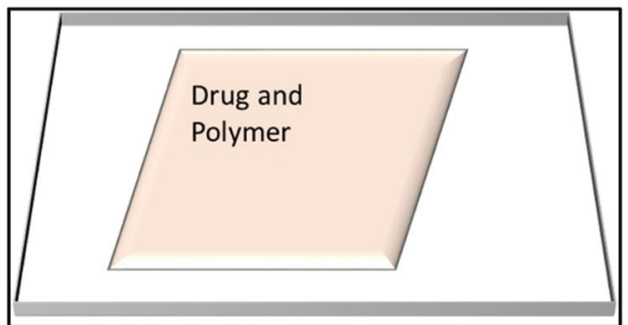

Polymeric nanosheet

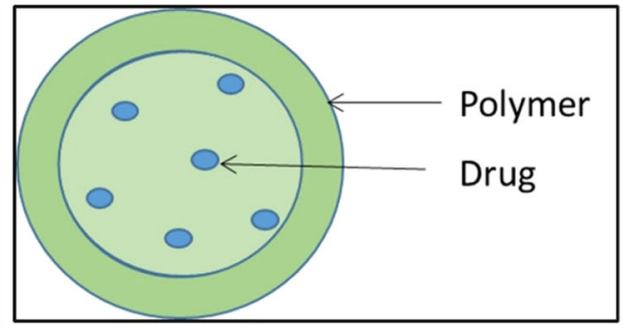

Polymeric nanoparticles

Fig. 3 Polymeric nanocarriers for delivery of TCS 
Dermal delivery of drugs in liposomal form was first reported by Mezei and Gulasekharam. Retention of triamcinolone acetonide (TA) in the epidermis and dermis using a liposomal lotion and gel with conventional lotion and gel of the free drug was compared. The results revealed that the liposomal gel had five times higher retention capability in the epidermis and three times higher in the dermis for the drug [40, 41]. Another study involving TA conducted by Clares et al. aimed at the preparation and characterization of multilamellar liposomes developed using a varying composition of L-alpha-phosphatidylcholine (PC), drug, different storage conditions, determining encapsulation efficiency and loss of drug. Stability studies showcased improved stability under refrigeration $\left(4-6{ }^{\circ} \mathrm{C}\right)$ (less early diffusion of drug through the liposomal wall) compared to those stored at room temperature. The addition of cholesterol in some formulations resulted in enhanced stability at refrigerated and room temperature conditions but also resulted in a decrease in average encapsulation efficiency than those formulated without cholesterol. Thus, antioxidants and/or preservatives were added to achieve acceptable vesicle dimensions, encapsulation efficiency, and enhanced stability [42].

Hydrocortisone acetate liposome was developed by Moldovan et al. using the film sonication technique. The formulation was characterized for drug content, particle size, and stability. The optimized batch with $0.2 \%$ drug concentration was incorporated as a carbopol-based gel. In vitro and in vivo release studies and skin blanching tests were performed. Both the in vitro and in vivo release study results displayed greater drug release from liposomal gel than that of conventional preparation. A fivefold reduction of drug dose was observed in the case of the liposomal gel than that of the marketed preparation. Thus, reducing the overall dosing frequency of the drug without decreasing its efficacy against dermal disorders [43].

Eroğlu et al. formulated a novel treatment method to decrease the problem of dermal targeting, which is observed after long-term corticosteroid therapy. The liposomes containing betamethasone valerate (BMV)/diflucortolone valerate (DFV) were developed by using the thin-film hydration method, which was further incorporated into the chitosan (CS)-based gel. Lecithin and CS NPs improve dermal targeting or increase the tolerability of drugs. Thus, BMV-and DFV-loaded liposomes were formulated and comparison was done with commercial creams and the developed NPs. Study results revealed that the drugs were localized in the SC and epidermis for both liposomes and NPs. In vivo studies performed on rats showed higher paw edema inhibition by liposome NP incorporated gel formulations than the commercial cream. The liposome in gels containing about ten times less drug than the commercial creams displayed comparable skin blanching effect. Dermatological scoring studies and TEWL studies of the formulation revealed better treatment efficacy of liposomes in $\mathrm{AD}$ induced rats than their commercial counterparts [44].

Proliposomes are ethanol solution-based or particulatebased preparations of phospholipid, which form liposomes after adding aqueous phase at suitable conditions such as phase transition temperature of the phospholipid selected. Khan, et al. investigated an innovative "slurry method" for the development of proliposome using sucrose carrier particles coated with cholesterol and soya PC in a 1:1 ratio. Beclometasone dipropionate was used as a model drug. Various ratios of lipid to sucrose carriers were utilized for the proliposome preparation and subsequently examined using scanning electron microscopy (SEM) for surface morphology and differential scanning calorimetry (DSC) for the determination of thermal properties. The proliposome was further subjected to hydration, and the prepared vesicles were compared with the conventional proliposome method, in terms of size of the formulated vesicle and drug entrapment efficiency. The SEM studies revealed that the lipids were uniformly coated with the sucrose carrier regardless of the lipid to carrier ratio. The novel resultant liposomes had a smaller median size than the conventional feed-line proliposome method. The DSC studies concluded that 50 mole\% beclometasone dipropionate drug was amorphous in the proliposome formulation and underwent crystallization on hydration, causing less drug entrapment. The liposomes were further centrifuged using deuterium oxide $\left(\mathrm{D}_{2} \mathrm{O}\right)$ as the dispersion medium; the beclometasone dipropionate entrapped vesicles precipitated out as a floating creamy layer, while some amount of the free drug sedimented as crystals. Liposomes generated from lipid to carrier ratio of 1:15 w/w using the slurry method showcased a higher entrapment efficiency as compared to the conventional feed-line proliposome method and liposomes formulated by the thin-film hydration method. Thus, this novel technique could potentially be explored for transdermal, pulmonary, and nasal drug delivery of beclomethasone dipropionate for various diseases [45].

Few examples of nonconventional liposomes present in the literature are reported below.

A nonconventional liposome was researched by Zhang et al. who fabricated a nanocomposite of betamethasone dipropionate (BDP) intercalated layered double hydroxide enclosed liposome by incorporating BDP molecules into sodium cholate micelles. The negatively charged BDPloaded micelles were further combined with positively charged layered double hydroxide single-layer nanosheets, forming a BDP/sodium cholate intercalated layered double hydroxide (BDP-Ch-LDH) guest-host nanohybrid. This nanohybrid was subsequently encapsulated with liposome by reverse evaporation method comprised of cholesterol and lecithin. These liposomes (BDP-Ch-LDH)-LS were 
characterized and found to exhibit less particle size and sedimentation rate compared to BDP-Ch-LDH. The results displayed the (BDP-Ch-LDH)-LS to have significant stability and water dispersity with improved sustained drug release than BDP-Ch-LDH. Hence, the use of liposome coating was found to be a successful approach for enhancing topical drug delivery [46].

Research by Rao et al. formulated clobetasol propionate (CP)-loaded liposome for intradermal drug delivery by lipid film hydration method using PC, cholesterol, $\alpha$-tocopherol. The liposomes were further incorporated into hydroxypropyl methylcellulose (HPMC) gel base and the final formulation was subjected to particle size analysis, surface morphology, in vitro drug release study, and skin blanching assay. The optimized liposomal gel batch containing 2:4:1 drug:PC:cholesterol ratio had a spherical multilamellar structure with a particle size of $5.13 \mu$, entrapment efficiency of $84.28 \%$.The gel also exhibited a low permeability coefficient, low diffusion rate and diffusion coefficient, and lesser skin blanching. Thus the liposomal gel-based system proved to be a good candidate for dermal drug targeting [47].

\subsubsection{Niosome}

Niosomes are bilayer vesicles made up of nonionic surfactants added to cholesterol with subsequent hydration in aqueous media. They were introduced by Handjani Vila et al. in 1979. Spans, Tweens, and Brij are the most commonly used surfactants used for niosomes preparation. Wasang ${ }^{\circledR}$, Gemini, and Bola surfactants are some rarely used surfactants. Niosomes are osmotically active and have higher oxidation stability than the other types of vesicles. They can entrap drugs with a wide solubility range and serve as a depot system for slow drug release. They are more stable, less immunogenic, and cheaper to formulate than phospholipids-based carriers as there is no need for expensive ingredients such as solubilizers like transcutol or edge activators like egg PC and bile salt [37, 39]. Some niosomal preparation reported in the literature is as discussed below.

Desoximetasone is a TCS used for the treatment of various localized skin conditions like $\mathrm{AD}$ and psoriasis as they decrease inflammation and itching. Shah et al. formulated the drug into a niosomal gel by using a systematic quality by design approach. The critical process parameters (mixing time and speed) and critical material attributes (surfactant, cholesterol amounts) were identified and used to interpret its effect on the critical quality attributes of topical niosome formulations. An organic-phase injection technique was employed to formulate the niosomes. The drug quantity, surfactant and cholesterol concentrations, and types of lipids were identified as the CMAs. While phase volumes, addition rate, temperature, and mixing time and speed were the CPPs. The entrapment efficiency, particle size distribution, PDI, and zeta potential were to determine the quality target product profile of niosomes. The critical impacting variables inferred from the experimental data for niosomes are as follows: surfactant and cholesterol concentrations, mixing parameters, and organic-phase addition rate. The desired desoximetasone niosomes had methanol: diethyl ether (75:25) as the organic system, drug:surfactant: cholesterol in 1:2:1 concentration, stearic acid as the chargeinducing material, $20 \mathrm{~mL}$ external phase and $10 \mathrm{~mL}$ internal phase volume, $65^{\circ} \mathrm{C}$ external phase temperature, $60 \mathrm{~min}$ mixing time, $650 \mathrm{RPM}$ mixing speed and $1 \mathrm{~mL} / \mathrm{ml}$ addition rate with optimum entrapment efficiency ranging from 63.90 to $95.58 \%$, particle size, and PDI ranging from 154.40 to $919.87 \mathrm{~nm}$ and 0.144 to 0.441 , respectively. Thus, comprehensive research on the understanding of drug product design for the formulation of dexamethasone noisome could be further incorporated into a suitable vehicle for topical application against skin conditions such as allergic reactions, $\mathrm{AD}$, and psoriasis [48].

$\mathrm{CP}$, a potent TCS, was formulated as a niosomal gel by Lingan et al. to extend the duration of action and minimize adverse effects. The niosomes were formulated by handshaking, thin-film hydration, and ether injection methods using varying ratios of cholesterol and nonionic surfactants (viz. span 40, 60, 80). The niosomes prepared with span 60 and cholesterol in a 1:0.5 ratio by thin-film hydration method showed a higher entrapment efficiency (91.37\%). It was further formulated as a carbopol-based niosomal gel. A comparative evaluation for drug content analysis, in vitro drug release, and in vivo pharmacodynamics was done for niosomal gel and marketed gel. Based on results, the authors concluded that niosomal gel for topical delivery of $\mathrm{CP}$ is a suitable approach than marketed preparation [49].

Sankar et al. formulated the niosomes of TA containing Span 20, cholesterol, cetrimide, and Brij 52 by thin-film hydration method using $2^{3}$ factorial design. The factorial design study revealed that cetrimide-based niosomal preparations exhibited desired particle size $(50-80 \mathrm{~nm})$ and higher skin permeability, entrapment efficiency, and in vitro drug release. The stability studies proved that the formulation was found to be stable for 90 days. Comparison between the niosomal formulation and marketed product for a clinical study conducted on healthy human volunteers using histamine wheal suppression test with and without iontophoresis displayed that the niosomal formulation with iontophoresis at $15 \mathrm{~min}$ has more effect on reduction in histamine wheal size than the marketed product with and without iontophoresis and niosomal formulation without iontophoresis than at 60 and 120 min when compared to the marketed product. These results confirm the ability of TA niosomes to permeate the skin effectively by noninvasive method iontophoresis thus proving its importance in the treatment of psoriasis as well as psoriatic arthritis [50]. 
Proniosomes, a novel drug delivery approach was used to design HC gel by Sankar et al. to increase drug permeation through the skin. The coacervation-phase separation method was used to prepare proniosome using varying ratios of lecithin, nonionic surfactants, and cholesterol. The span: span combinations (span 20:span 80, span 20:span 60, and span 20:span 40) exhibited better entrapment than the combinations of span:tween (span 20:tween 80, span 20: tween 60, span 20:tween 40). The proniosome formulation of $1 \%$ HC containing span 20:span 80 showed improved in vitro drug release $(58.29 \%)$ in comparison with other proniosome formulations. Higuchi and Peppas plot showed diffusion type release of proniosome $\mathrm{HC}$ gel. Comparison of the in vivo studies in mice among the proniosome $1 \%$ $\mathrm{HC}$ formulation and commercially marketed $1 \% \mathrm{HC}$ cream proved that the former was more active than the conventional cream. Thus, the topical application of $\mathrm{HC}$ in the form of proniosomes prolonged drug action and its permeability [51].

\subsubsection{Ethosomes}

Ethosomes are vesicles made of phospholipids, alcohol (ethanol) in high concentrations (20-45\%), and water. Ethanol acts as a permeation enhancer and its interdigitization effect also provides flexibility to the vesicle structure and helps them squeeze through the skin pores. The presence of ethanol also helps to entrap drugs of different physiochemical properties. First introduced by Touitou et al. in 2000, these vesicles may be unilamellar or multilamellar in structure with a size range of $30 \mathrm{~nm}$ to a few microns [37, 52].

$\mathrm{CP}$ is a widely used antipsoriatic agent and thus enhances its dermal penetration ability. Korade et al. formulated CP ethosomal gel composed of a fixed quantity of soya lecithin, propylene glycol, and water. A varying quantity of ethanol was used for the preparation of the ethosomes and was further formulated as carbopol-based gel formulations were evaluated for entrapment efficiency, in vitro drug release, skin irritation study, physical and rheological test for gel. The optimized formulation had a higher quantity of ethanol with a vesicular size of $110 \mu \mathrm{m}, 74.2 \%$ drug entrapment efficiency, spherical vesicular shape, and displayed maximum drug release at $6 \mathrm{~h}$, which was higher than the conventional marketed gel [53].

In a recent study Akhtar et al. formulated nanoethosomal glycolic lipid vesicles of TA for AD therapy by the infusion method. The study analyzed the effect of binary solvents on topical delivery of TA by the development of the vesicles by the infusion method. Gels formulation (TA10) containing soya lecithin $(2 \% \mathrm{w} / \mathrm{v})$, ethanol $(40 \% \mathrm{w} / \mathrm{v})$, and propylene glycol $(10 \% \mathrm{w} / \mathrm{v})$ showed higher in vitro permeation. Confocal Laser Scanning Microscopy studies confirmed uniform and deep penetration of the drug into the epidermis. The combination of ethanol and propylene glycol resulted in enhanced penetration enhancement. Thus, nanoethosomal glycolic vesicles signified as an effective approach for topical delivery of TA [54].

\subsubsection{Transferosomes}

Cevc and Blume developed transferosome in 1992, which is the type of deformable vesicular system composed of edge activators enclosed within a phospholipid matrix, which when applied on the skin surface squeeze through intercellular spaces of the SC under the influence of the transepidermal water-activity gradient. High deformability is observed due to the accumulation of edge activator molecules at the site of high stress, due to their raised propensity for curved structures leading to vesicle penetration through the skin. Transferosomes can entrap both low and high molecular weight drugs and protect them from enzymatic metabolic degradation. They can be used as a depot formulation for controlled topical as well as systemic delivery of drugs. Compared to liposomes, transferosomes can reach intact deeper regions of the skin after topical administration delivering higher drug concentrations for transdermal applications [55]. They do possess disadvantages such as expensive nature, chemical unstable, and purity of phospholipids, which acts as an important criterion to be considered while manufacturing the vesicles $[37,56]$.

The authors Cevc and Blume characterized the efficacy of $\mathrm{HC}$ and dexamethasone transferosome with a dose ratio of 1:50 as observed in the vasoconstriction test. The rate of transferosome skin penetration, cutaneous drug biodistribution, and drug bioefficacy study was conducted. The results suggested a three- to fivefold enhancement in HC potency and a decrease in the minimum effective dose of $\mathrm{HC}$ transferosome was observed to be $2-3 \mu \mathrm{g} \mathrm{cm}^{-2}$ (as compared to creams and lotions i.e., $>10 \mu \mathrm{g} \mathrm{cm}^{-2}$. A twofold prolonged suppression of drug-induced edema was observed in the $\mathrm{HC}$ transferosome delivery. Dexamethasone effective dose in murine skin reduced more than ten times when delivered in transferosome formulation and a decrease in the minimum effective dose of dexamethasone transferosome was observed to be $<0.1 \mu \mathrm{g} \mathrm{cm}^{-2}$, which suppress arachidonic acid-induced murine ear edema by more than $>50 \%$ on average. The effective dose of dexamethasone was reduced more than ten times as compared to cream and lotion [57]. They also examined the biodistribution and murine ear edema of TA transferosomes by in vivo test using radioactive label measurements. The required dose of TA transferosome is also significantly reduced. Suppression of 75\% of arachidonic acid-induced murine ear edema for at least $48 \mathrm{~h}$ was observed at a dose of $0.2 \mu \mathrm{g} \mathrm{cm}^{-2}$. The resultant transferosome showed a tenfold decrease in drug 
dose than conventional dosage forms. Thus, TCS transferosomal formulation improved the dermal targeting and therapeutic risk-benefit ratio of both the drugs [58].

Gillet et al. displayed the ability to enhance the aqueous solubility of betamethasone by complexing it with HP $\gamma$ cyclodextrin to form an inclusion complex-based transferosomes using the film evaporation method. The formulation was composed of soybean PC or dimyristoylphosphatidylcholine (DMPC) and sodium deoxycholate as edge activator were compared to classical non-deformable liposomes. PC-based transferosome had better vesicle size, deformability, and encapsulation efficiency than DMPC transferosome. The use of sodium deoxycholate increased encapsulation efficiency up to 1.8 times and up to 1.3 times the drug diffusion percentage in comparison with classical liposomes. The transferosomes also demonstrated good stability at $4{ }^{\circ} \mathrm{C}$ thus proving to be successful tools for cutaneous drug delivery [59].

In another study, Shashi et al. formulated dexamethasone-loaded tranferosomal gel by modified handshaking technique using a fixed concentration of drug $(50 \mathrm{mg})$ and a varied concentration of lecithin:tween 80. The transferosomal gel was further prepared by utilizing a cold method using a combination of carbopol and sodium alginate (combined ratio- $0.5 \% \mathrm{w} / \mathrm{w}$ ) as a gel base. The resultant transferosomal gel was further characterized for entrapment efficiency, particle size, zeta potential, and polydispersibility, SEM, spreadability, viscosity, and in vitro drug release. The formulated dexamethasone transferosomes showed a high entrapment efficiency from 71.6 to $94.63 \%$. SEM and optical microscope were used to examine surface morphology of the formulations, which displayed spherical vesicles with sizes ranging from 82.23 to $170.6 \mathrm{~nm}$ and PDI ranging from 0.126 to 0.259 which indicated a homogenous population of vesicles distribution. The viscosity of the formulations was observed in the range of 31000-31500 cps with a spreadability of $12.17-16.63 \mathrm{~g}$ $\mathrm{cm} / \mathrm{s}$. The in vitro drug release study suggested that the optimized formulation (lecithin:tween $80=2: 1$ ) displayed a sustained release action and a high percentage of drug entrapment efficiency, which helped to improve patient compliance of the formulation [60].

\subsubsection{Ufasomes}

Ufasomes are also known as unsaturated fatty acid vesicles. They are composed of suspensions of closed lipid bilayers (fatty acids) oriented in such a way that their hydrocarbon tails are directed toward the membrane interior and the carboxyl groups are in contact with water, and their ionized species (soap), which are restricted to $\mathrm{pH}$ range of 7-9. The stability of ufasomal preparation depends on the proper selection of fatty acid, amount of cholesterol, buffer, $\mathrm{pH}$ range, amount of lipoxygenase, and the presence of divalent cations [61]. Ufasomes enhance drug penetration into the skin through the SC by attaching to it and allowing lipid exchange between the outermost layers of the SC. Ufasomes are more stable and cheaper than liposomes. They have better entrapment efficiency for both hydrophilic and hydrophobic drugs [39].

Dexamethasone-loaded ufasomal gel was formulated and characterized by Mittal et al. for evaluating antiinflammatory activity for cutaneous drug delivery by carrageenan-induced rat paw edema model. Ufasomal suspension of dexamethasone was prepared by the sonication technique using varying concentration ratios of Span 80, Span 20, and cholesterol. The carbopol-940-based ufasomal gel formulation was prepared by the hydration method. The formulation was further characterized for vesicle size, shape, particle size distribution, drug entrapment efficiency, zeta potential, in vitro drug release, SEM, Fourier transform infrared spectroscopy (FTIR), in vitro, and ex vivo skin permeation studies, in vivo anti-inflammatory activity, and stability studies. The vesicles formed were spherical and had a multilamellar structure under TEM. The formulation formed with an 8:2 molar ratio of drug:oleic acid was observed to have a greater number of vesicles and higher drug entrapment efficiency. This ufasome was further optimized and evaluated. The dermal partitioning and transdermal permeation of optimized formulation were significantly higher $(P<0.05)$ than plain drug and plain gel formulation owing to the addition of surfactant, which acted as a permeation enhancer. The optimized ufasomal gel formulation displayed skin permeation of about 4.7 times greater than plain gel containing a drug. The antiinflammatory activity carried out using the carrageenaninduced rat paw edema model showcased a significant reduction of edema $(P<0.10)$ compared to that of the commercial product. The oleic acid-based vesicles penetrate intact skin and form depots. Thus, it could be used as an alternate carrier for topical drug delivery. Based on the results, the authors concluded that drug encapsulation in ufasomal gel can serve as a potential carrier for the delivery of anti-inflammatory drugs for dermal drug delivery [62].

\subsubsection{Solid lipid nanoparticles (SLN)}

SLN is solid vesicles containing lipids, lipid-like materials, or a mixture possessing a diameter ranging in the range of 10 $\mathrm{nm}$ to $10 \mu \mathrm{m}$. They were first developed in the early 1990s in Germany and Italy. They can be formulated by high-pressure homogenization, ultrasonication technique, etc. They offer an opportunity to formulate hydrophobic and hydrophilic drug moieties into a suitable dosage form for controlled drug release to the skin and enhance the stability of compounds from light and oxygen. They are also biodegradable, 
biocompatible, cost-effective, simple, and suitable for industrial applications. The disadvantages of SLNs include limited encapsulation efficiency and drug loading due to drug solubility in lipid, structure, crystallinity, polymorphic forms of lipids, and high water content [63]. These are some SLN formulations reported in the literature.

TA was successfully formulated as SLN by Pradhan et al. [64] by using the emulsification-ultrasonication approach. This study also dealt with the optimization of various critical process parameters such as surfactant, lipid, and drug concentration along with homogenization, sonication time, and homogenizer speed. The maximum TA solubilization capacity was observed in Compritol $^{\circ} 888$ ATO. The effect of variables like concentrations of lipid, surfactant, and drug as well as sonication time on the formulation properties was observed. The results revealed an increasing particle size was obtained with higher concentrations of lipid and drug whereas, an increase in sonication time resulted in a decrease in particle size. The prepared SLNs showed prolonged drug release compared to a pure drug suspension. A substantial amount of drug was observed into the epidermal layer of skin in the in vitro skin distribution of drug-loaded SLN suspension. Thus, the authors conclude that the developed SLN system of TA demonstrates the systemic escape of drug after administration, which might eliminate side effects due to systemic exposure [64].

Pradhan et al. [65] developed fluocinolone acetonide (FA)-loaded SLN by emulsification-ultrasonication technique. Ex vivo study revealed drug release up to $12 \mathrm{~h}$ and in vitro drug release study of FA-loaded SLN suspension showed a substantial amount of FA on the epidermal layer of the skin whereas plain FA suspension displayed relatively very less amount of drug in the epidermis and dermis. Thus, FA-loaded SLN could be established as effective drug delivery for the treatment of psoriasis [65].

Bikkad et al. formulated halobetasol propionate (HP)loaded SLN (HP-SLN) for enhanced dermal targeting and controlled drug release. HP-SLN was prepared by the solvent injection technique using glycerol monostearate as solid lipid and Tween 80 as a stabilizer. Glycerol monostearate-based SLN dispersions having a particle size of $200 \mathrm{~nm}$ and entrapment efficiency of $93 \%$ were selected as the optimized batch and subsequently incorporated into the carbopol-based gel. HP-SLN-loaded gel showed prolonged drug release up to $12 \mathrm{~h}$ in an ex vivo study. In vitro drug deposition and skin irritation studies of HP-SLN formulation displayed better accumulative uptake of the drug and nonirritant when compared to marketed formulation. These results signify SLNs as a promising system for topical delivery of HP in dermal therapy [66].

Madan et al. prepared mometasone furoate (MF)-based SLN by a solvent injection method using glycerol monostearate as a solid lipid. The optimized batch showed sustained drug release (more than $8 \mathrm{~h}$ ) and maximum entrapment (up to 55.59\%). The prepared SLN-loaded gel showed 15.21 times and $83.52 \%$ higher skin permeability and skin deposition, respectively, than the marketed cream formulation. About 20 times higher skin deposition was observed in SLN-loaded gel than plain drug-loaded gel. Thus the production of MF-loaded SLNs gel could be a more efficacious, cost-effective, and commercially viable alternative to conventional gels [67].

Zhang et al. developed an SLN of BMV for extended and localized delivery by solvent injection method for the treatment of eczema or psoriasis. The reservoir formation of the drug was investigated on human skin using static Franz diffusion cells. Monostearin SLN showed greater drug reservoir formation and lower permeation rate in the human epidermis than commercial lotions and drug suspensions. The SLN formulation containing beeswax did not decrease the drug permeation through the skin and no increase in drug content was observed in the upper layers of the skin. Therefore, it was concluded in the study that depending on the SLN composition, they can act as a potential carrier to target BMV to the inflammatory site of the skin while diminishing the detrimental systemic adverse effects [68].

Kalariya et al. formulated CP-loaded SLN (CP-SLN) in cream base by using high-pressure homogenization technique and characterized for mean particle size, surface morphology, and percent drug entrapment. The optimized CPSLN was smooth and spherical under SEM; with an average particle size of $177 \mathrm{~nm}$ and $92.05 \%$ drug entrapment. The formulation and marketed cream were further evaluated for in vitro drug release study, drug permeation, and skin uptake studies via Franz static diffusion cell across human cadaver skin. CP-SLN reported a 1.9- and 1.2-fold reduction in the degree of inflammation and itching, respectively, against marketed cream. Thus CP-SLN was observed to have an improved therapeutic response for topical antipsoriatic drug delivery [69].

Schlupp et al. studied the influence of the drug-particle interaction of SLN on penetration enhancement of three glucocorticoids-prednisolone, the diester prednicarbate, and BMV. SLNs were prepared by the high-pressure homogenization technique. It was composed of $10 \%$ Compritol 888 ATO, 2.5\% Poloxamer 188, and $0.1 \%$ of glucocorticoids. Glucocorticoids-particle interaction, drug release, and skin penetration were evaluated. Both with SLN and cream, the prednisolone release was superior to diester prednicarbate, which exceeded BMV release. Skin penetration study results did not display a change in the rank order with the cream, thus proving that it is predominantly influenced by drug release. The penetration profile for the glucocorticoids is independent of release and the physiochemical properties of the glucocorticoids when 
applying SLN dispersions, indicating the specific interaction of carrier and skin surface lipids being of high importance. Loaded SLNs completely changed, and differences between the steroids were almost lost. SLNs influence skin penetration by an intrinsic mechanism linked to the lipid nature and nanosize of SLN and thus help in epidermal drug targeting [70].

Cavalli et al. formulated $\mathrm{HC}$ inclusion complex-based SLN of $\mathrm{HC}$ and progesterone were formed with $\beta$-cyclodextrin $(\beta \mathrm{CD})$ and 2-hydroxypropyl- $\beta$-cyclodextrin. The inclusion complexes were prepared by the coprecipitation technique. SLN was formulated by a microemulsion-based method using stearic acid, soya PC, glycerol monostearate, taurocholate sodium salt, butanol, and distilled water. The formation of the complexes was confirmed by DSC analysis. The presence of inclusion complexes did not significantly increase the size of SLN; it remained below $100 \mathrm{~nm}$. Drug release results revealed higher drug release of $\mathrm{HC}$ than progesterone. Aqueous solubility of the system was also increased than regular drug-loaded SLN. While free drug-loaded SLN showed faster drug release than steroid $\beta$ CD SLN. Therefore these modified liposomal nanocarrier systems could serve as a tool for controlled dermal drug delivery [71].

\subsubsection{Nanostructured lipid carrier (NLC)}

NLCs are second-generation SLN constituting a fluid lipid phase spatially blended into a solid lipid matrix or confined at the surface of solid platelets and surfactant layer. The lipid spatial structure leads to enhanced drug loading, controlled drug release, and improved stability, and lesser water content in comparison to SLN. NLCs have been extensively been used in dermatotherapeutics. Some applications of antidermatitis and antipsoriatic NLC drug delivery of TCS have been elaborated in detail [72, 73].

Pradhan et al. [74] formulated FA-loaded NLC using a modified microemulsion method comprising of Compritol 888 ATO, Miglyol ${ }^{\oplus} 812$, and drug dissolved in organic solvent of methanol and acetone $(1: 1, \mathrm{v} / \mathrm{v})$ in a water bath at $70{ }^{\circ} \mathrm{C}$ and varying concentration of polysorbate 80 as the aqueous phase. In vitro skin permeation studies showed a significantly higher amount of FA deposited in the epidermal and dermal layer of skin by FA-loaded NLC suspension in comparison to plain FA suspension. Thus, FAloaded NLC can act as a promising nanocarrier system for psoriasis management [74].

Pradhan et al. [75] also fabricated TA-loaded NLCs for effective psoriasis management. A lipid excipient Compritol ${ }^{\oplus} 88$ ATO and medium-chain triglyceride Miglyol $\$ 12$ were used for the formulation of TA-loaded NLCs by modified microemulsion-ultrasonication technique. The observations of in vitro skin distribution showed a significant quantity of TA on the epidermis when treated with TA-loaded NLCs suspension. Thus, decreasing the occurrence of adverse side effects and acting as an effective antipsoriatic nanoformulation system [75].

HP is a potent corticosteroid used in dermal inflammatory conditions. Recently, Carvajal-Vidal et al. formulated HP-loaded NLCs by high-pressure homogenization technique. The formulation is comprising of a drug, surfactant, glyceryl distearate, and capric glycerides. The formulation was characterized and an optimized batch with particle size below $200 \mathrm{~nm}$, containing $0.01 \%$ of drug and $3 \%$ of total PDI $<<0.2$ and an encapsulation efficiency $>>90 \%$ was selected. The in vitro and in vivo tests revealed that cell cultures of THP-1 and cultured human keratinocyte cells (HaCaT) showed a significant reduction in IL-8 production in the presence of HB-NLC thus proving its efficacy as a potential tool for dermatological therapy [76].

Silva et al. investigated the epidermal targeting potential of CP by formulating it as NLC and CS-coated NLC. The NLC was prepared by the microemulsion method using stearic acid, oleic acid, lecithin, and sodium taurodeoxycholate along with drug molecules. Whereas, clobetasolloaded nano-lipid carriers were coated with CS of 0.072, 0.145 , and $0.220 \% \mathrm{w} / \mathrm{v}$ concentrations. Epidermal targeting and skin permeation studies were performed using Franz diffusion cell on the SC and the whole epidermis. Improved skin permeation was noticed with drug-loaded NLC containing a one-fifth dose of CP compared to that of the marketed formulation. Epidermal drug retention for both CS-coated and uncoated NLC was more than 81-fold higher than the commercial formulation. Conclusively, NLCs can be administered for targeted epidermal drug delivery and decreasing adverse side effects of drugs [77].

\subsubsection{Nanoemulsion}

Nanoemulsions are nontoxic, nonirritant isotropic, transparent/translucent heterogenous dispersed systems comprising of two immiscible phases-oil and water phase. They exist as an oily system (2-20\%w/w) dispersed in an aqueous system $(\mathrm{O} / \mathrm{W})$ droplet, or an aqueous system dispersed in an oily phase (W/O) droplets. It is stabilized by an interfacial layer of emulsifiers and co-emulsifiers [78]. The effect of zeta potential plays a significant role in the stability of such systems. For single emulsifier system carrying a low molecular weight surfactant zeta potential of more than $\pm 30 \mathrm{mV}$ provides good stability and it gets excellent when it reaches toward $\pm 60 \mathrm{mV}$. In the case of zeta potential $\pm 20 \mathrm{mV}$ or more short-term stability is observed. A surface charge of the -5 to $+5 \mathrm{mV}$ range indicates fast aggregation of the globules thus causing instability of the system. In the case of high molecular weight surfactants, a shift to a higher magnitude of zeta potential is observed to maintain globules 
in Brownian motion for a longer duration and impart stability to the system [35]. The droplet size of these emulsion systems is of the order of $20-400 \mathrm{~nm}$. Although emulsion systems are considered thermodynamically unstable, the tiny droplet size of nanoemulsion contributes to physical stability against creaming, coalescence, and sedimentation. Instability is often caused by Ostwald ripening effect and sudden changes in temperature and $\mathrm{pH}$. Nanoemulsions are prepared by three methods mainly: high-pressure homogenization, microfluidization, and phase-inversion temperature. Incorporation of both hydrophobic and hydrophilic in nanoemulsions is possible in the form of W/ $\mathrm{O}$ or $\mathrm{O} / \mathrm{W}$ nanoemulsions [79-81]. Some topical TCS nanoemulsion formulations are discussed below.

Prednicarbate is a new TCS extensively used in AD therapy. Baspinar et al. designed a stable positively charged nanoemulsion system for prednicarbate drug delivery. Optimal concentrations of phytosphingosine were used to induce a positive charge and help damaged skin restoration. A highpressure homogenization technique was employed to prepare the formulation. The optimization of batches was done based on the influence of parameters like production temperature, type of homogenizer, homogenization cycles, pressure, and concentration of oil phase, emulsifiers, and phytosphingosine. The results thus revealed that nanoemulsion was formulated with $0.6 \%$ phytosphingosine, $20.0 \%$ Eutanol, and $2.0 \%$ Lipoid E80 and Tween 80 and it should be developed under elevated temperatures, low homogenization pressures, and higher numbers of homogenization cycles (e.g., $300 \mathrm{bar}$ and 10 cycles) for a physically and chemically stable positively charged nanoemulsion system [82].

BDP has anti-inflammatory action and is used as an effective agent for mild-to-moderate psoriasis therapy. BDP was successfully investigated and evaluated for the production of the nanoemulsion system by Alam et al. The nanoemulsion was formulated using an aqueous phase titration method with eucalyptus and babchi oil as the oil phase. The nanoemulsion system with Tween 20 surfactant was observed to be physically and chemically stable with a shelf life of 2.64 years at room temperature. The nanoemulsion was further incorporated into carbopol-based gel and evaluated for in vitro skin permeation studies, and antiinflammatory studies by carrageenan-induced paw edema to prove the efficacy of the system. Thus, a safe and effective nanoemulsion gel formulation of BDP provided enhanced permeation of the drug, reduced dosing frequency, and sustained the drug release for a longer duration of time, and also have improved anti-inflammatory action [83].

Sajid et al. formulated BMV nanoemulsion for antidermatitis therapy. The aqueous phase titration method was employed for nanoemulsion preparation with sefsol as the oil phase and tween 20 surfactants, transcutol co-surfactant, distilled water as the components of the aqueous phase. The optimized formulation was converted to carbopol 934-based hydrogel. The hydrogel formulation was evaluated for in vivo anti-inflammatory, in vivo nickel-induced contact dermatitis activity, and in vivo irritation study. The results indicated an insignificant irritation score of 1.83. Inhibition of inflammation for nanoemulsion gel and marketed cream to be 84.2 and $45.05 \%$, respectively. Nickel-induced dermatitis study confirmed no stimulation of inflammatory, or immune response was observed due to the nanoemulsion gel formulation of BMV [84].

Alam et al. investigated the antidermatitis potential of CP nanoemulsion gel. The nanoemulsion was prepared by aqueous phase titration method using algal oil as the oil phase, tween 20, and PEG 200 as surfactant and co-surfactant, respectively, and distilled water forming the aqueous phase. The optimized nanoemulsion batch was incorporated into a hydrogel vehicle using carbopol-971 having a viscosity of $97.57 \pm 0.04 \mathrm{PaS}$. In vivo antiinflammatory showed inhibition of inflammation by the drug-loaded nanoemulsion gel $(84.55 \%)$ compared to and placebo formulations $(41.04 \%)$. Contact dermatitis revealed higher NTPDase activity (anti-inflammatory action) in the treatment with the CP-loaded nanoemulsion in comparison to that of placebo nanoemulsion gel [85].

\subsection{Polymeric nanocarrier system}

Polymeric nanocarrier-based formulations are submicrometric colloidal drug carrier systems. They have diverged composition and can be categorized as nanocapsules, NPs, nanosheets, nanofibers, etc. [86]. Some of the polymeric nanocarriers are depicted in Fig. 3.

\subsubsection{Polymeric nanocapsule}

Nanocapsules are nanostructured polymeric capsules wherein a drug molecule is enclosed in a pocket within a polymeric matrix or shell. These formulations exhibit the potential to enhance skin permeation and drug release rates across the skin. Polymeric nanocapsules prevent drug degradation, and these formulations can be applied topically by incorporation into semisolid vehicles like hydrogels and emulgel. Extensive research has been conducted to scrutinize the probability of the use of nanocapsular formulation for dermatitis and psoriasis therapy [87, 88]. Lipid-core nanocapsules (LNC) are colloidal-sized vesicular systems comprising a mixture of solid lipids and liquid lipids below $40{ }^{\circ} \mathrm{C}$. However, classical nanocapsules are comprised of only liquid oil. Polymeric nanocapsules have numerous applications viz. sustained drug release, specific tissue targeting, etc. [86].

Fontana et al. designed CP-loaded LNC hydrogel. Sorbitan monostearate, poly( $\varepsilon$-caprolactone) (PCL), polysorbate 
80 were utilized for the preparation of nanocapsule, and Carbopol Ultrez $10 \mathrm{NF}$ was used for the preparation of hydrogel. In vivo efficacy studies conducted on Wistar rats by nickel sulfate-induced dermatitis method revealed prolonged drug release from prepared hydrogel than that of commercial preparation [89].

MF is a synthetic heterocyclic TCS used as an antipsoriatic agent owing to its anti-inflammatory properties. Melero et al. formulated Momentasone furoate-lipid-core nanocapsules (MF-LNC) by a self-assembling method using PCL as the polymer and oil mixture of caprylic triglycerides. Carbopol ${ }^{\oplus}$ Ultrez was used as a gelling agent to develop semisolid formulations. Skin permeation and penetration studies indicated slower drug release from MFLNC hydrogel than nonencapsulated drug-loaded hydrogel. The developed formulation controls its skin permeability without significantly changing its SC accumulation. Further, the amount of drug penetrating the deeper skin layers was also controlled. Therefore, MF-loaded LNC is a promising approach for the treatment of skin diseases with the minimization of systemic drug absorption [86].

Beber et al. formulated cationic polymeric nanocapsulebased hydrogel encapsulating dexamethasone in its cavity. Eudragit RS 100 was utilized as the polymer to prepare the batches. The cationic polymeric nanocapsules fabricated showed zeta potential of $+11.38 \pm 1.7 \mathrm{mV}$, the average particle size of $139 \pm 3.6 \mathrm{~nm}$, and the encapsulation efficiency of $81 \pm 2 \%$. The prepared hydrogel displayed acidic $\mathrm{pH}$, plastic behavior, and non-Newtonian flow. The optimized gel showed sustained drug release and boosted epidermal drug deposition and retention. Thus, dexamethasone-loaded cationic polymeric nanocapsule hydrogel can be used as a promising nanocarrier system for antipsoriatic therapy [90].

Desonide is a non-fluorinated synthetic TCS that has been extensively for 30 years in dermatotherapeutics. Recently, Antonow et al. fabricated desonide-loaded nanocapsule suspension by utilizing polymers like Eudragit S100 and Eudragit L100 and desonide-loaded LNC. Interfacial deposition of the preformed polymer was the technique employed for the production of nanocapsule suspension. The optimized formulation had negative zeta potential and average particle size in the range of particle size $161-202 \mathrm{~nm}$ and $\mathrm{PDI}<0.20$. In vitro drug release study was also conducted, which showed monoexponential (Desonide LNC and free desonide) and biexponential (desonide LNC and desonide nanocapsule suspension) release profile, regardless of the type of drug release method. A sensitive HPLC method was developed and validated for the quantification of desonide in the nanocapsule formulation. Thus, a nanoencapsulation form of desonide was successfully designed capable of minimizing corticosteroid adverse effects and improving its efficacy [91].

\subsubsection{Polymeric micelle}

Polymeric micelles are polymeric NPs with a particle size of 5-100 nm fabricated from amphiphiles, above the critical micelle concentration with a "core-corona" structure. Micelles create a core for hydrophobic drug moiety, which is surrounded by a hydrophilic rigid shell. Micelles enhance drug bioavailability, increase drug-loading capacity, reduce drug degradation, and decrease adverse effects [73]. Research work presenting polymeric micelles for antidermatitis and antipsoriatic drug delivery is as follows.

In a recent study, Aseem et al. formulated beclomethasone dipropionate polymeric micelle intended for the treatment of AD. The thin-film hydration technique was employed for micelle preparation. Concentrations of pluronic L121 with either poloxamer P84 or pluronic F127 with varying surfactant mixture-to-drug ratios were used. A full factorial design (FFD- $3^{2} \cdot 2^{1}$ ) was selected for optimization of various variables like pluronic L121 concentration range with either pluronic F127 or poloxamer P84 and surfactant mixture-to-drug ratios. Beclomethasone dipropionate polymeric micelles with higher entrapment efficiency, zeta potential, smaller particle size, and PDI were prepared. Two formulae one with pluronic L121/poloxamer P84 mixture and another using pluronic L121/pluronic F127 mixture were selected for ex vivo skin deposition studies. The optimized formula with the highest dermal deposition of $541.76 \pm 20.71$ was further subjected to TEM and physicochemical stability studies and an HPMC-based hydrogel was formulated. A comparative study of the viscosity and dermal drug deposition between the prepared hydrogel and the marketed Beclozone ${ }^{\circledast}$ cream was conducted. In vivo histopathological analysis of the mixed micelle hydrogel and Beclozone demonstrated successful sub-chronic dermatitis treatment in an animal model by the prepared hydrogel within (6 days) as compared to Beclozone (12 days), thus ensuring enhanced patient compliance and lesser adverse effects [92].

\subsubsection{Polymeric nanospheres/microspheres}

A polymeric nanosphere is a matrix type, solid colloidal particle in which drugs are dissolved, entrapped, encapsulated, chemically bound, or adsorbed to the polymer matrix. These nanospheres with a diameter of 100 and $200 \mathrm{~nm}$ are larger and more polydispersible than micelles. These nanospheres are formulated by two methods namely, emulsification/solvent evaporation and nanoprecipitation technique [93]. Whereas microspheres are spherical microscopic particles that range in size from 1 to $1000 \mu \mathrm{m}$ or beyond prepared by using the solvent evaporation method, spray drying technique, suspension, emulsion, dispersion, and sedimentation polymerization method [94]. 
Reported research work in literature for TCS-loaded polymeric microsphere for antipsoriatic drug delivery is explained in detail.

CP-loaded-poly(D,L-lactic-co-glycolic acid) (PLGA) microspheres were developed by Badilli et al. using oil-inwater emulsion solvent evaporation technique. The formulation was characterized for particle size analysis, morphological characterization, DSC, and XRD analyses. In vitro, drug release studies carried out on emulgel formulations containing pure drug and drug-loaded microspheres and commercial cream products reported higher drug release from emulgel formulations than commercial products. The microsphere with 1:5 drug/polymer ratio and homogenization speed and time of $8000 \mathrm{rpm}$ and time $1 \mathrm{~min}$, respectively, was considered as the optimized formulation batch. The delay in the drug release due to encapsulation of the drug in the microparticle also causes the prolonged duration of action and reduced side effects during topical drug delivery [95].

\subsubsection{Nanosponges/microsponges}

Nanosponges/microsponges are tiny polymeric porous microspheres capable of entrapping drugs within their sponge-like cavities. Nanosponges are of particle size of $<100 \mu \mathrm{m}$ while microsponge is of the particle size of 5-300 $\mu \mathrm{m}$. These systems are prepared predominantly by methods such as liquid-liquid suspension polymerization technique, hyper cross-linked cyclodextrin polymers, ultrasound-assisted synthesis, and quasi-emulsion solvent diffusion [96, 97]. Research work presenting polymeric microsponges for TCS drug delivery for skin ailments is as follows.

Betamethasone microsponge gel was formulated by Mohanty et al. using a quasi-emulsion solvent diffusion method using varying concentrations of Eudragit RS 100 as the polymer. The microsponges were evaluated for morphology, drug-excipient compatibility, encapsulation efficiency, and in vitro drug release. The SEM analysis proved that the prepared microsponges were spherical and porous. FTIR studies revealed no incompatibility among drugs, excipients, and optimized formulation. The encapsulation efficiency of the optimized microsponge was found to be $64.6 \%$ with in vitro drug release that was found to be $77 \%$ in $8 \mathrm{~h}$. The optimized microsponge batch was further incorporated into a carbopol-based gel evaluated for $\mathrm{pH}$ and in vitro release. The in vitro drug release of the gel was found to be $73 \%$ in $8 \mathrm{~h}$. The $\mathrm{pH}$ of the gel was reported to be suitable for topical application $\mathrm{pH}$ 6.8. Thus a controlled release topical microsponge gel was formulated for the treatment of dermatitis [98].

MF is a non-fluorinated, synthetic, TCS, indicated for use against psoriasis. Rekha et al. formulated MF entrapped microsponges by emulsion solvent diffusion method. Drug-excipient compatibility studies were evaluated by FTIR analysis. Surface morphology, production yield, loading efficiency, and surface morphology of microsponges were also analyzed. The results revealed that an increase in the drug:polymer ratio caused a reduction in the drug release rate. Cumulative drug release from microsponge after $8 \mathrm{~h}$ ranged from 78 to $95 \%$ with an entrapment efficiency maximum of $86.13 \%$. Hence a microsponge-loaded MF formulation was prepared and evaluated for controlled release action against various dermatological conditions [99].

Devi et al. fabricated and evaluated CP-based microsponge gel prepared by employing a quasi-emulsion solvent diffusion method using Eudragit RS 100 as the polymer. The prepared microsponges possessed particle size in the range $12.2-45.80 \mu \mathrm{m}$, entrapment efficiency of $60.00-96.37 \%$, and drug release of 60.60-92.82. Optimized CP microsponge batches were incorporated into carbopol gel base, and in vitro release of microsponge was compared to free drug-loaded gel. The microsponge formulation followed zero-order kinetics indicating a constant rate of drug release with no initial burst release. In vivo, antipsoriatic activity studies using the mice tail model revealed greater therapeutic efficacy of microsponge gel in comparison to free drug-loaded gel. Hence, microsponge-based nanocarrier system extended drug release reduced systemic side effects and enhanced therapeutic activity [100].

D'Souza et al. formulated FA entrapped microsponge to reduce the systemic side effects of drugs and promote controlled drug delivery to the skin. The quasi-emulsion solvent diffusion method was employed for the preparation of the microsponge. Drug-excipient compatibility was analyzed by FTIR and DSC. Surface morphology, production yield, loading efficiency, and particle size analysis revealed that the spherical porous microsponge powder had a diameter of 31.34-82.26 $\mu \mathrm{m}$ and loading efficiency of 86-90\%. The microsponges were then incorporated into carbopol 934 gels and analyzed for drug release and comparative anti-inflammatory studies. Drug release of about 22.44 was observed for $2 \mathrm{~h}$ thus promoting the release of drugs in a controlled fashion for topical drug delivery [101].

\subsubsection{Nanofiber}

Nanofibers are extensively utilized as topical drug delivery systems for implants, wound healing, cosmetics, electrospun sutures, etc. while nanobeads are utilized for oral, injectable, and ophthalmic formulations. TA is an effective TCS used against inflammatory skin conditions. In the following study, Jahangiri et al. formulated TA-loaded PLGA nanofibers by electrospraying technique. DSC and $\mathrm{X}$-ray powder diffraction displayed reduced drug 
crystallinity during the electrospraying process. Dissolution tests exhibited faster drug release for pure drug and physical mixtures compared to the nanoformulations. Thus, a prolonged-release triamcinolone nanofiber formulation was successfully examined for exploiting its anti-inflammatory potential [102].

\subsubsection{Nanosheet}

Nanosheets are ultra-thin (thickness in the nanometer order), two-dimensional nanostructures with high drugloading capacity, transparency, flexibility, and adhesiveness. They have greater aspect ratios $\left(>10^{9}\right)$ based on the surface area, resulting in variable bulk state physicochemical and electronic characteristics [103, 104].

Hatanaka et al. assessed the potential for application of nanosheets for transdermal drug delivery by fabricating BMV biocompatible polymeric nanosheets using poly(Llactic acid) or poly(lactic-co-glycolic) acid via spin-coatingassisted layer-by-layer technique by utilizing sacrificial membrane. In comparison to conventional ointment, these nanosheets absorb and deliver a higher quantity of BMV by controlling the quantity of drug added, polymers used, and controlled release membranes. The duration of application of these sheets to any area of skin can be prolonged owing to its optimum thickness, high transparency, high adhesiveness, and good moisture permeability. These properties are observed to be retained even in the presence of drugs thereby presenting novel, transdermal polymeric nanosheets for various dermal disorders [105].

\subsubsection{Polymeric NPs}

Polymeric NPs are prepared from biocompatible and biodegradable polymers as well as synthetic polymers, which allow an increase in drug bioavailability, better drug release over time, target specificity, reduced toxicity, and undesirable effects [106, 107].

4.2.7.1 CS NPs CS is a natural linear cationic polysaccharide formed by the deacetylation of chitin, which is the fundamental constituent of arthropod exoskeletons. It consists of randomly distributed $\beta$-(1-4)-linked Dglucosamine and its $\mathrm{N}$-acetylated derivative of CS. It is extensively used for developing sustained release systems for nasal, ophthalmic, transdermal, and implantable devices [107, 108]. A detailed overview of some CS NPs is enlisted below (Table 3).

Hussain et al. prepared HC encapsulated polymeric NPs with a focus on enhancing drug transport. Ex vivo drug permeation study demonstrated decreased flux and permeation coefficient in $\mathrm{NC} / \mathrm{Nga}$ mouse. Higher aggregation of a drug on the skin was detected in comparison to the control groups. In vivo study revealed that the nanoparticulate carrier system had controlled TEWL, erythema intensity, dermatitis index, and increased skin thickness. Histopathological investigation revealed inhibition of the elastic fibers fragmentation and fibroblast infiltration thus proving the anti-fibrotic and anti-inflammatory activity of NPs against $\mathrm{AD}$ lesions. The research hence concluded that the HCloaded NPs provided remarkable therapeutic benefits against AD (Hussain et al. [109]).

Özcan et al. prepared DFV-loaded lecithin/CS NPs by ionic interaction technique to optimize the efficiency of DFV and maintain drug localization in skin layers. The NPs were fabricated using positively charged polysaccharide CS and negatively charged lipidic lecithin by supramolecular self-organizing interaction approach. The optimized batches were further incorporated into CS gel. The results of in vivo anti-inflammatory and skin blanching activities comparable to that of commercial cream. Thus, the results of in vitro and in vivo results demonstrated that the lecithin/CS NPs incorporated into CS gel could be a potential approach for skin delivery of DFV in psoriasis therapy [110].

In another study, Özcan et al. formulated BMV-loaded poly (lactide-co-glycolide) (PLGA) and lecithin/CS NPs. PLGA NPs were developed using emulsion-diffusion-evaporation technique while the lecithin/CS NPs were prepared by ionic interaction between negatively charged lipid lecithin and positively charged CS. Both the drug-loaded PLGA and lecithin-CS NPs were incorporated into CS gel for further evaluation. Drug accumulation in skin layers from both gel formulations was observed to be increased compared to the commercial formulation. Both the formulations significantly enhanced anti-inflammatory and skin blanching properties although they possess ten times less quantity of BMV than marketed cream. Moreover, TEWL measurement revealed no barrier function variations after the application of NPs on the skin. 1.58-Fold increment in the epidermal concentration of BMV by lecithin/CS NPs compared to PLGA NPs. Thus, lecithin/CS NPs were selected as a suitable candidate for topical drug delivery in the treatment of inflammatory dermal disorders [111].

Şenyiğit et al. formulated lecithin/CS NPs by direct injection of soybean lecithin alcoholic solution comprising $\mathrm{CP}$ into an aqueous solution of CS. This nanoparticulate suspension was incorporated into a CS gel. Carrageenaninduced hind paw edema studies on Wistar rats were performed to evaluate the anti-inflammatory activity of formulations. The results of Carrageenan-induced hind paw edema studies performed on Wistar rats indicated that NPbased gel formulation possesses higher anti-inflammatory activity and TEWL measurements revealed insignificant skin barrier function disturbance for the nanoformulation. Thus, it was concluded that the use of lecithin/CS NPs in CS gel proved to be more efficacious as compared to 


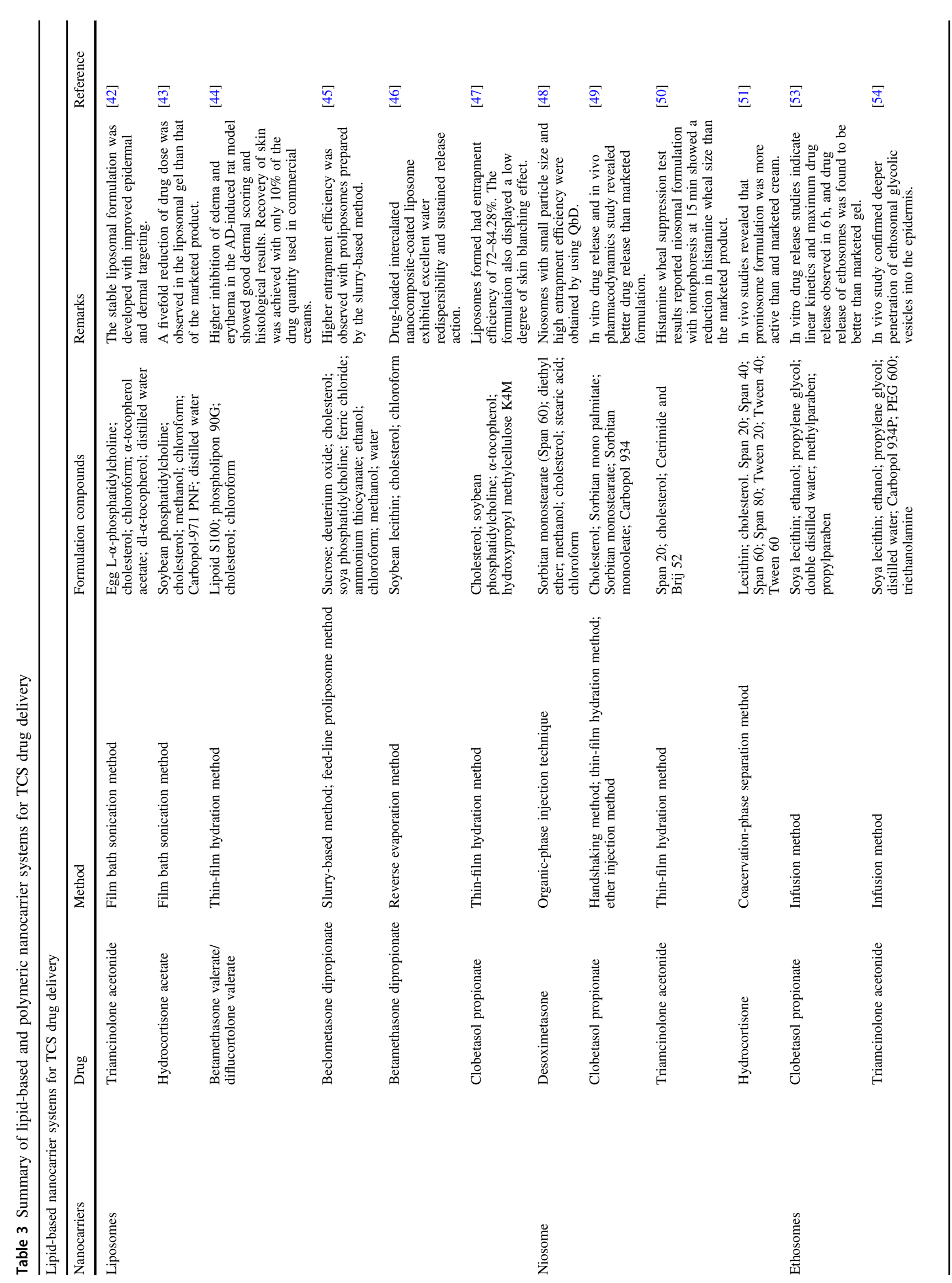


88 Page 20 of $29 \quad$ Journal of Materials Science: Materials in Medicine (2021) 32:88

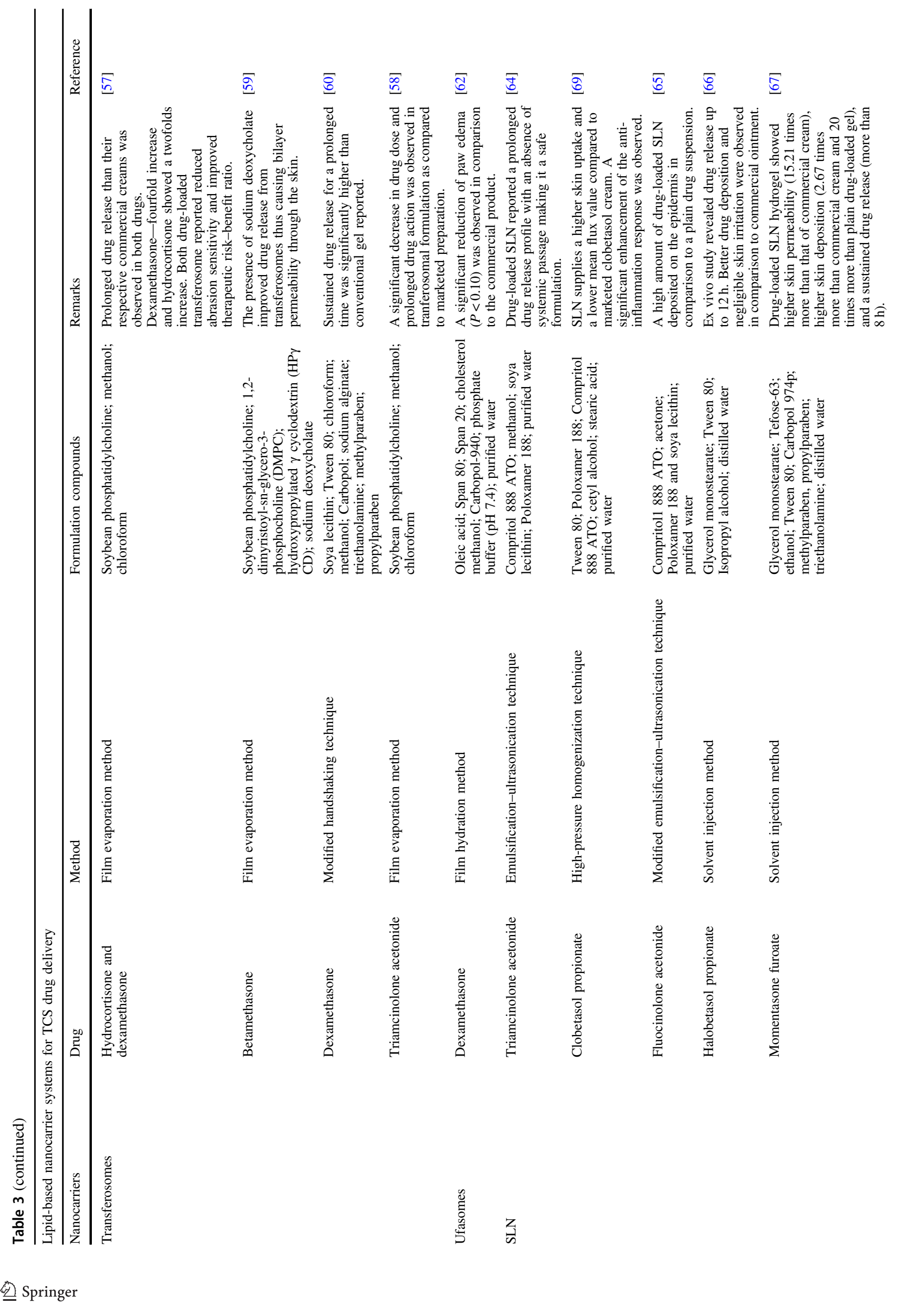




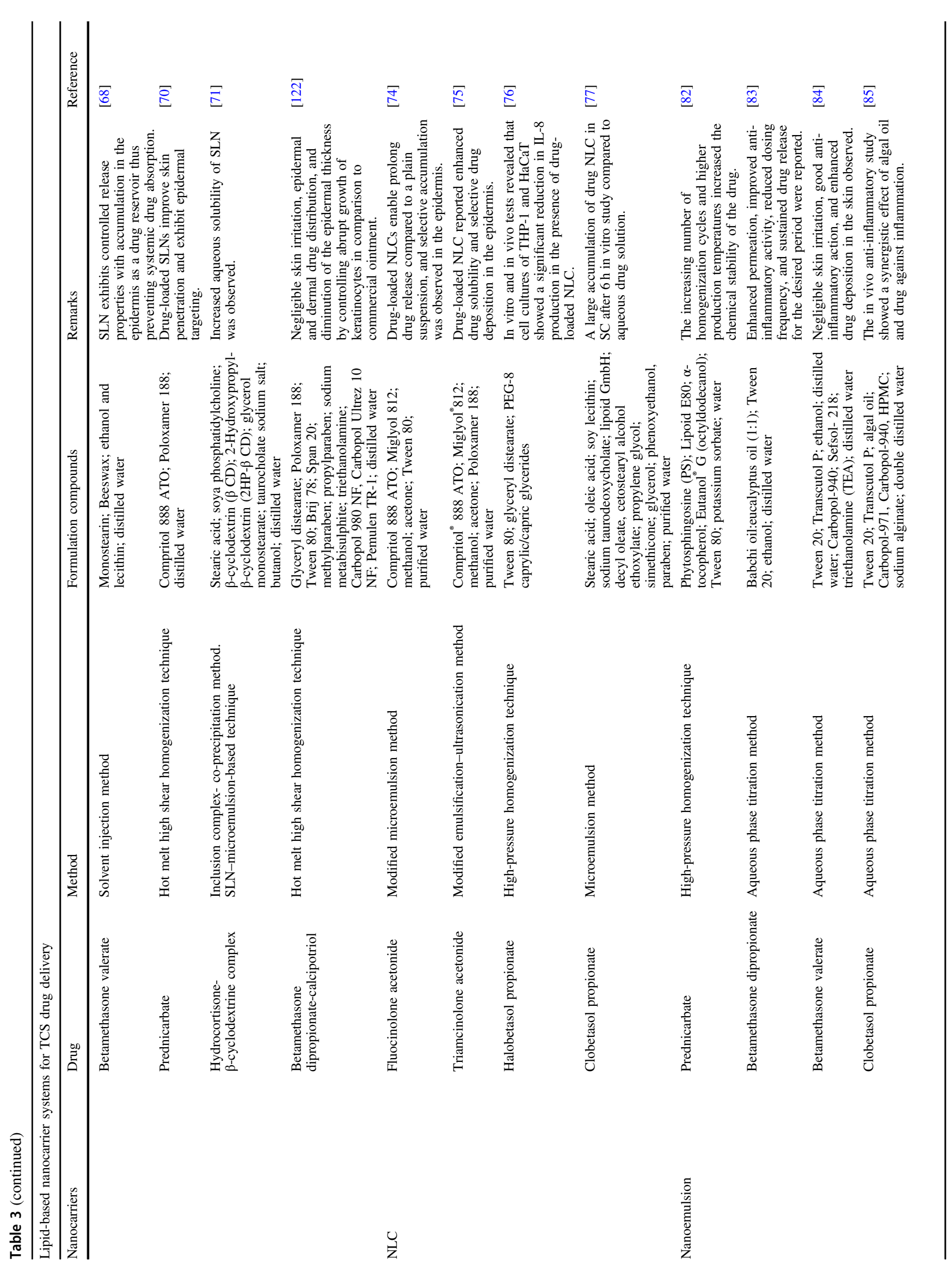




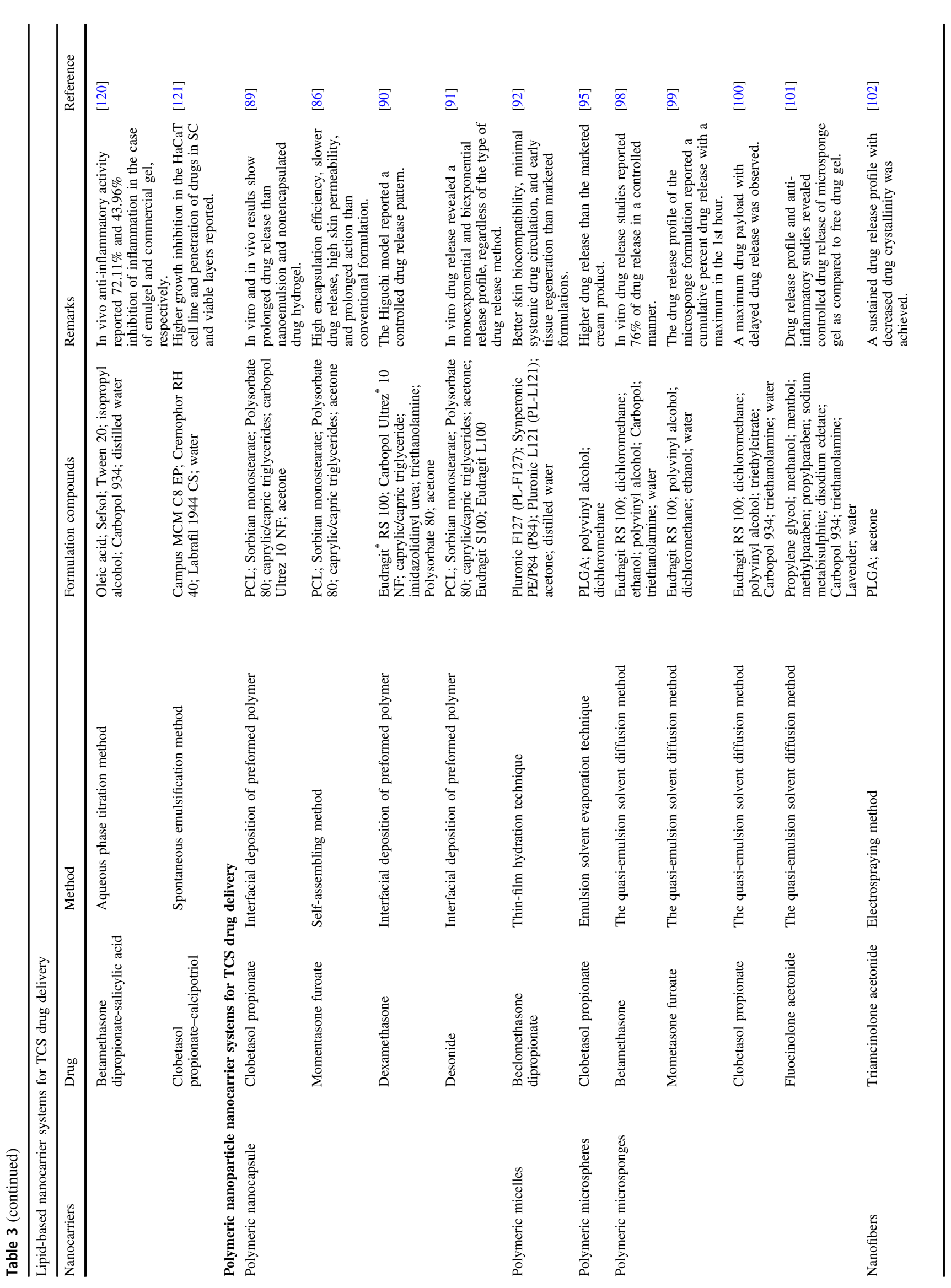




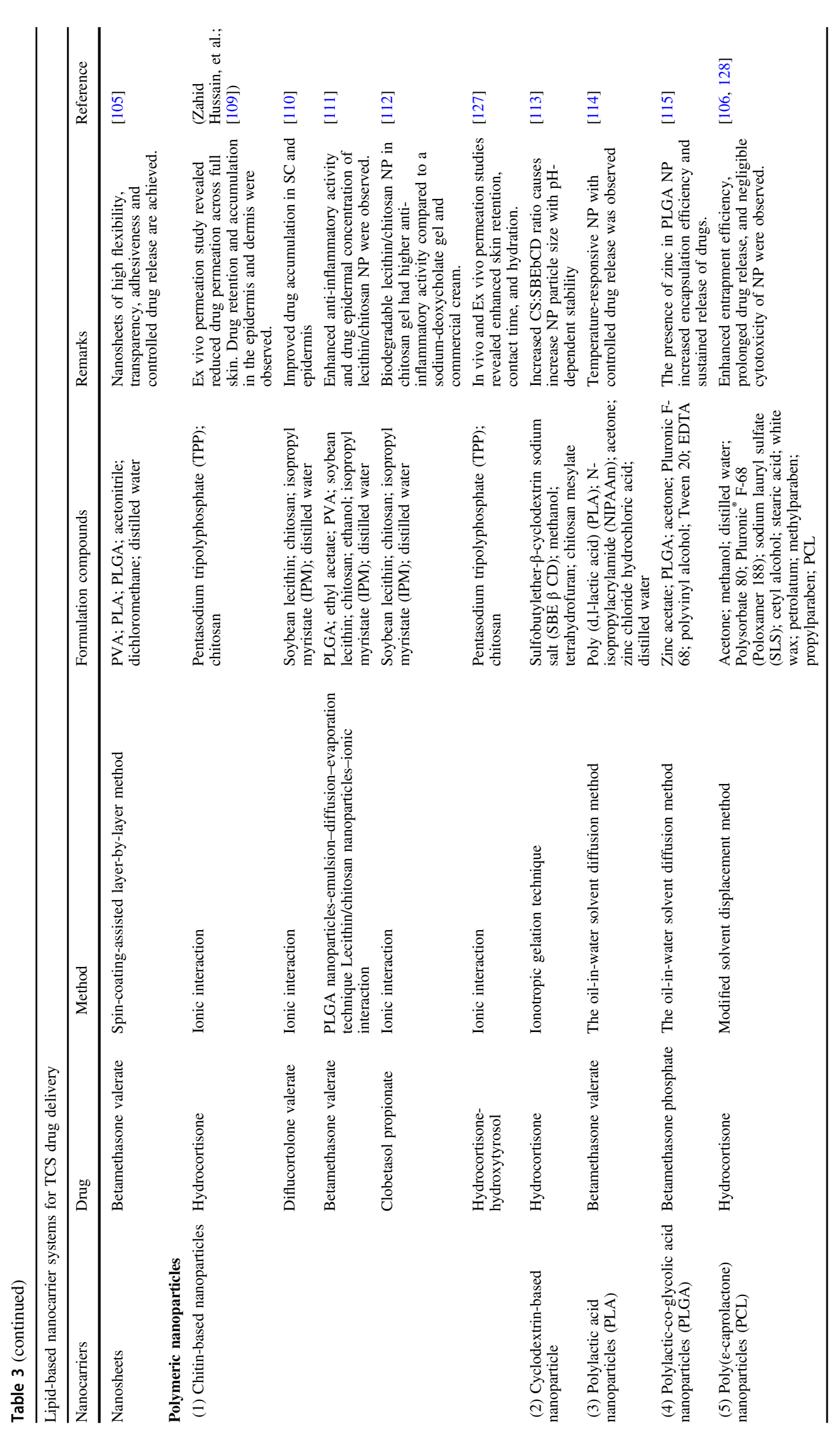


conventional sodium-deoxycholate gel and commercial cream formulations of $\mathrm{CP}$ [112].

4.2.7.2 Cyclodextrin-based NP Fülöp et al. fabricated a polymer/cyclodextrin NP with $\mathrm{CS}$, and sulfobutylether$\beta$-cyclodextrin (SBE- $\beta$-CD). The NPs were produced by the ionotropic gelation technique and comprised of low molecular weight $(10 \mathrm{kDa})$ cationic $\mathrm{CS}$ polysaccharide crosslinked with polyvalent anions (SBE- $\beta-\mathrm{CD})$. The lipophilic nature of the cyclodextrin cavities helped in the encapsulation of poorly water-soluble lipophilic drugs like HC (aqueous solubility of $0.3 \mathrm{mg} / \mathrm{ml}$ ). The particle diameter ranged from 200 to $1000 \mathrm{~nm}$ and the drug release rate was regulated through $\mathrm{CS}: \mathrm{SBE}-\beta-\mathrm{CD}$ molar ratio. The encapsulation ability drug in the NPs decreased with increasing CS concentration. Ionic cross-linking can also provide improved encapsulation even at low initial SBE- $\beta-C D$ and CS concentrations. The stability of the formulation depends on $\mathrm{pH}$ and is highly stable in acidic conditions but becomes unstable at $\mathrm{pH}$ above 7 . This proves that the CS/SBE- $\beta$-CD$\mathrm{NP}$ can be used as a $\mathrm{pH}$-controlled drug delivery carrier for dermal inflammatory conditions [113].

4.2.7.3 Polylactic acid nanoparticles (PLA) A biodegradable temperature-responsive drug delivery system was developed by Ayano et al. by the O/W solvent diffusion approach in the presence of zinc ion using a blend of PLA and PNIPAAm-PLA. BMV was further encapsulated in this PLA homopolymer and block copolymer blend. Intracellular drug uptake was analyzed using murine macrophagelike cell line, to analyze the cellular uptake of fluorescent PLA/PNIPAAm-PLA NPs at 30 and $37^{\circ} \mathrm{C}$. Below the lower critical solution temperature $\left(34^{\circ} \mathrm{C}\right)$, cellular uptake was not observed. The biodegradable temperatureresponsive NPs exhibited favorable intracellular drug uptake and thermo-responsive drug release [114].

\subsubsection{Polylactic-co-glycolic acid nanoparticles (PLGA)}

Ishihara et al. developed BDP-loaded poly(d,l-lactic/glycolic acid) (PLGA) or poly(d,1-lactic acid) (PLA) NPs for targeted sustained release of corticosteroid. NPs were formulated using the $\mathrm{O} / \mathrm{W}$ solvent diffusion method with PLGA (or PLA), zinc, drug, and surfactant. The particle size and encapsulation efficiency of the NP-loaded drug were influenced by factors such as PLGA/PLA concentration and the quantity of zinc added. NPs were formulated with $8 \%$ w/w drug content and particle diameter ranging from 80 to $250 \mathrm{~nm}$. The release rate of BDP from NPs was gradual and released for at least 8 days while BDP-loaded NPs showed release of the drug after only 2 days. Glycolic/lactic acid ratio and molecular weight of PLGA or PLA were the two factors that influenced the release rate of the drug from the nanoparticle. Thus incorporation of zinc elevates the encapsulation efficiency of the drug in nanoparticles and induces sustained drug release from the nanoparticles for dermatotherapy [115].

4.2.7.5 PCL nanoparticles Rosado et al. fabricated and characterized HC-loaded PCL-based nanoparticles to enhance drug release and diminish associated adverse effects. Controlled drug release and significant difference between permeation of nano encapsulated and free $\mathrm{HC}$ was revealed by in vitro drug release study. Further, in vitro toxicity analysis reported no signs of toxicity after nanoencapsulation. Thus, the nanoencapsulation of $\mathrm{HC}$ could demonstrate quicker control over $\mathrm{AD}$ with negligible steroidal side effects [106].

\subsection{Metallic nanoparticles}

Metallic nanocarriers are extensively used for drug delivery and diagnostics owing to their small size, high surface area, surface modification property, and high reactivity towards living tissues [73]. Typically, researched metallic nanoparticles for TCS loading are silica nanoparticles and metalorganic framework nanoparticles.

\subsubsection{Silica nanoparticles}

Ghasemnejad et al. fabricated hexagonal mesoporous silica nanoparticles (SBA-15) modified with (3-aminopropyl) triethoxysilane for betamethasone sodium phosphate delivery onto the skin. Physicochemical characterization of the synthesized and modified SBA-15 was performed using elemental analysis by CHN techniques, N2 adsorptiondesorption isotherm, FTIR spectroscopy, and surface morphology analysis. Maximum loading of $33.69 \%$ was accomplished under the optimized condition of $\mathrm{pH}: 1.8$, time: $3.54 \mathrm{~h}$, and drug/silica ratio: 1.7 . The developed formulation was found to be an excellent carrier for corticosteroid therapy via the topical route [116]. Silica nanoparticle as one of the metallic nanoparticles is shown in Fig. 4.

\subsubsection{Metal-organic framework nanoparticles}

Li et al. formulated magnetic metal-organic frameworks-101 (MOF-101) functionalized with graphite-like carbon nitride material $\left(\mathrm{Fe}_{3} \mathrm{O}_{4} / \mathrm{g}-\mathrm{C}_{3} \mathrm{~N}_{4} / \mathrm{MIL}-101\right)$ prepared by chemical coprecipitation method for the efficient enrichment of glucocorticoids in cosmetics. The nanocarriers were prepared by the co-precipitation technique with the incorporation of five corticosteroid drugs such as $\mathrm{HC}$, dexamethasone, fluocinonide, desonide, and flunisolide in the cosmetic along with other polycyclic aromatic hydrocarbons and benzene compounds. MIL-101 is a MOFs material with Lewis acid potential, used for the enrichment of trace substances in complex samples. 


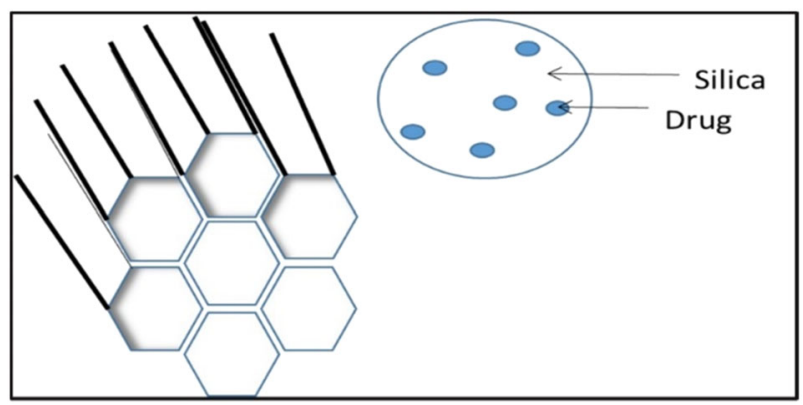

Fig. 4 Silica nanoparticle-based delivery system for TCS

The open metal sites are occupied by water molecules, which decreases its enrichment effect. The immobilized carbon materials on the surface of MOFs act as a hydrophobic barrier for the water molecules to occupy the open metal sites of MIL101 and thus improving the material enrichment effect. Enhanced selectivity toward glucocorticoids was observed due to the hydrogen bonding effect with $\mathrm{g}_{-} \mathrm{C}_{3} \mathrm{~N}_{4}$ and the sizematching effect with MIL-101 than polycyclic aromatic hydrocarbons and benzene compounds. $\mathrm{Fe}_{3} \mathrm{O}_{4} / \mathrm{g}-\mathrm{C}_{3} \mathrm{~N}_{4} / \mathrm{MIL}$ 101 material also acted as adsorbents in magnetic solid-phase extraction coupled with ultra-performance liquid chromatography-mass spectrometry, which was used for extraction of drug from the sample. Good water stability, better molecular selectivity, and higher extraction efficiency of all the five drugs were observed thus these metal-organic frameworks can be employed for drug delivery for topical application [117].

\section{Peptide-drug conjugate (PDC)}

PDCs is a carrier for targeted drug delivery wherein one or more drug molecules is attached to a short peptide chain through a biodegradable linker. The self-assembling potential of small-molecule peptides helps in the formation of these nanosized peptide-drug amphiphiles. This arena of research is extensively studied for drug delivery in cancer therapy but its scope in the case of topical drug delivery is underexplored [118]. The recent progress made in the designing of corticosteroid-loaded PDC for antipsoriatic action is hereby explained in detail.

A pH-responsive biodegradable poly-L-glutamic acid (PGA)-FA conjugate was synthesized and characterized by Dolz-Pérez which allowed controlled drug release to alleviate skin inflammation. The conjugate was prepared by the addition of hyaluronic acid PGA cross polymer vehicle for permeation enhancement and conjugating drug to the peptide via a pH-responsive ester linker. In vitro anti-inflammatory studies, in vivo psoriasis mouse model, and ex vivo human skin permeation studies analysis of the conjugate revealed inhibition of pro-inflammatory cytokine release, epidermal drug targeting, and reduced dermal drug accumulation. Thus PGAFA conjugate represents an effective drug delivery system for effective topical psoriasis therapy [119].

\section{Combination therapy}

Combination corticosteroid therapy employed between TCS and other classes of drugs like antibacterial agents, retinoids, keratolytic or vitamin D3 analogs provides a synergistic effect against dermatitis and psoriasis therapy. Many antipsoriatic and antidermatitis conventional combination therapy formulations are commercially available and extensively used in dermatotherapeutics. The nanoemulsion-based gel has been formulated for many effective combinations like BDP-salicylic acid [120] and CP-calcipotriol [121]. These formulations displayed lower toxicity, sustained release action, and better anti-inflammatory activity than their conventional marketed counterparts. Recently, Sonawane et al. formulated BDP and calcipotriol-loaded SLN using hot melt high shear homogenization technique. Glyceryl distearate was employed as a solid lipid-based for the preparation of SLN for both drugs. These SLN of both drugs were further integrated into carbopol-based gel for topical drug delivery. In vitro studies like skin permeation, dermal distribution, the antiproliferative effect on $\mathrm{HaCaT}$ cells, and in vivo studies like Draize patch irritation, TEWL, and antipsoriatic mousetail studies experiments were performed to test the antipsoriatic potential of the prepared formulation. The in vitro drug release studies displayed restricted permeation to the epidermal and dermal layer of skin. Draize patch test and TEWL study exhibited no skin irritation and enhanced skin tolerance of SLN. A decline in the keratinocyte growth rate was demonstrated by the in vitro $\mathrm{HaCaT}$ cell line study, while a comparative in vivo mouse-tail model study between the formulated SLN gel and commercial Daivobet ointment showed a significant increase in the melanocyte count and reduction in the epidermal thickness with the use of SLN gel than that of the marketed formulation. Thus, the successful in vitro and in vivo results solidify the potential for the launch of marketed nanocarrier-based combination therapy products after successful clinical trials in humans [122].

Polymeric nanocarrier systems have also been investigated for combination therapy against AD. Siddique et al. developed a HC and hydroxytyrosol (HT) CS nanoparticle by ionic crosslinking of CS with pentasodium tripolyphosphate ions. HT is a polyphenolic component of olive oil with a potent antimicrobial and antioxidant activity, which acts in improving the efficacy of the nanoparticle against AD. HC-HT CS nanoparticles of particle size $235 \pm 9 \mathrm{~nm}$ and zeta potential $+39.2 \pm 1.6 \mathrm{mV}$ were integrated into a cream and evaluated for dermal irritation and repeated dose toxicity test HC-HT CS nanoparticle displayed 100 -fold higher LD 50 values than the 
normal human dose of hydrocortisone in comparison with the marketed preparation. HC-HT CS nanoparticles were observed to be nonirritant and in vivo analysis revealed that nanoencapsulation diminished the adverse effects of $\mathrm{HC}$ thus acting as a well-tolerated alternative for $\mathrm{AD}$ therapy [123].

\section{Conclusion}

Currently, the market is flooded with conventional TCS preparations extensively used for psoriasis and AD. The use of current TCS therapy for dermatological conditions are inadequate because of their local and systemic toxicity, low benefitto-risk ratio, reduced treatment efficacy (due to lower penetration of active in the viable epidermal region and poor retentive capability), and high dosing frequency with poor patient compliance [124]. Developments in nanotechnologybased drug delivery have successfully opened avenues for improved drug targeting, better therapeutic value, and reduced toxicity for transdermal therapy of psoriasis and AD. Regardless of the immense research work conducted on formulation development and preclinical studies, very few clinical studies have been conducted to establish the clinical safety of such novel nanocarrier-based systems. Nonetheless, future research must focus on evaluating the benefit-to-risk ratio for the drugs included in nanocarriers. Thus, nanocarrier-based topical drug delivery for corticosteroid would eventually become a crucial accession to dermatotherapy offered for $\mathrm{AD}$ and psoriatic patients in the near future $[6,125]$.

\section{Compliance with ethical standards}

Conflict of interest The authors declare no competing interests.

Publisher's note Springer Nature remains neutral with regard to jurisdictional claims in published maps and institutional affiliations.

Open Access This article is licensed under a Creative Commons Attribution 4.0 International License, which permits use, sharing, adaptation, distribution and reproduction in any medium or format, as long as you give appropriate credit to the original author(s) and the source, provide a link to the Creative Commons license, and indicate if changes were made. The images or other third party material in this article are included in the article's Creative Commons license, unless indicated otherwise in a credit line to the material. If material is not included in the article's Creative Commons license and your intended use is not permitted by statutory regulation or exceeds the permitted use, you will need to obtain permission directly from the copyright holder. To view a copy of this license, visit http://creativecommons. org/licenses/by/4.0/.

\section{References}

1. Roy P, Saha SK, Paul PC, Reza AK, Nandi AK, Sultana S, et al. Effectiveness of topical corticosteroid, topical calcineurin inhibitors and combination of them in the treatment of vitiligo. Mymensingh Med J. 2016;25:620-7.

2. Lee A, Fischer G. Diagnosis and treatment of vulvar lichen sclerosus: an update for dermatologists. Am J Clin Dermatol Springe Int Publ. 2018;19:695-706.

3. Roberts MS, Mohammed Y, Pastore MN, Namjoshi S, Yousef S, Alinaghi A, et al. Topical and cutaneous delivery using nanosystems. J Control Release. 2017;247:86-105.

4. Marwah H, Garg T, Goyal AK, Rath G. Permeation enhancer strategies in transdermal drug delivery. 2014;7544:1-15.

5. Khurana B, Arora D, Narang R. Topical delivery of nanoemulsion for antipsoriatic drugs. J Drug Deliv Ther. 2018;8:1-11.

6. Akhtar N, Verma A, Pathak K. Exploring preclinical and clinical effectiveness of nanoformulations in the treatment of atopic dermatitis: Safety aspects and patent reviews. Bull Fac Pharm, Cairo Univ. 2017;55:1-10.

7. Palmer BC, DeLouise LA. Nanoparticle-enabled transdermal drug delivery systems for enhanced dose control and tissue targeting. Molecules. 2016;21:7-9.

8. Dommasch ED, Li T, Okereke OI, Li Y, Qureshi AA, Cho E. Risk of depression in women with psoriasis: a cohort study. Br J Dermatol. 2015;173:975-80. https://doi.org/10.1111/bjd.14032.

9. Baviera G, Leoni MC, Capra L, Cipriani F, Longo G, Maiello N, et al. Microbiota in healthy skin and in atopic eczema. BioMed Res Int. 2014;436921.

10. Nutten S. Atopic dermatitis: global epidemiology and risk factors. Ann Nutr Metab. 2015;66:8-16.

11. Seykora J, Dentchev T, Margolis DJ. Filaggrin-2 barrier protein inversely varies with skin inflammation. Exp Dermatol. 2015;24:720-2.

12. Furue M. Regulation of filaggrin, loricrin, and involucrin by IL4, IL-13, IL-17A, IL-22, AHR, and NRF2: pathogenic implications in atopic dermatitis. Int J Mol Sci. 2020;21:1-25.

13. Nakahara T, Kido-Nakahara M, Tsuji G, Furue M. Basics and recent advances in the pathophysiology of atopic dermatitis. J Dermatol. 2021;48:130-9.

14. Bergmann S, von Buenau B, Vidal-y-Sy S, Haftek M, Wladykowski E, Houdek P, et al. Claudin-1 decrease impacts epidermal barrier function in atopic dermatitis lesions dose-dependently. Sci Rep. 2020;10:2024

15. Karczewski J, Dobrowolska A, Rychlewska-Hańczewska A, Adamski Z. New insights into the role of T cells in pathogenesis of psoriasis and psoriatic arthritis. Autoimmunity. 2016;49:435-50.

16. Helmick CG, Lee-Han H, Hirsch SC, Baird TL, Bartlett CL. Prevalence of psoriasis among adults in the U.S.: 2003-2006 and 2009-2010 National Health and Nutrition Examination Surveys. Am J Prev Med. 2014;47:37-45.

17. Navarini AA, Burden AD, Capon F, Mrowietz U, Puig L, Köks $\mathrm{S}$, et al. European consensus statement on phenotypes of pustular psoriasis. J Eur Acad Dermatol Venereol. 2017;31:1792-9.

18. Higgins E. Psoriasis. Medicine. 2017;45:368-78.

19. Lowes MA, Suárez-Fariñas M, Krueger JG. Immunology of psoriasis. Annu Rev Immunol. 2014;32:227-55.

20. Kim J, Krueger JG. Highly effective new treatments for psoriasis target the IL-23/Type $17 \mathrm{~T}$ cell autoimmune axis. Annu Rev Med. 2017;68:255-69.

21. Chiricozzi A, Pitocco R, Saraceno R, Nistico SP, Giunta A, Chimenti S. New topical treatments for psoriasis. Expert Opin Pharmacother. 2014;15:461-70.

22. Harden JL, Krueger JG, Bowcock AM. The immunogenetics of psoriasis: a comprehensive review. J Autoimmun. 2015;64:66-73.

23. Guttman-Yassky E, Krueger JG. Atopic dermatitis and psoriasis: two different immune diseases or one spectrum? Curr Opin Immunol. 2017;48:68-73.

24. Rendon A, Schäkel K. Psoriasis pathogenesis and treatment. Int J Mol Sci. 2019;20:1475. 
25. Brezinski EA, Dhillon JS, Armstrong AW. Economic burden of psoriasis in the United States a systematic review. JAMA Dermatol. 2015;151:651-8.

26. D'Souza LS, Payette MJ. Estimated cost efficacy of systemic treatments that are approved by the US Food and Drug Administration for the treatment of moderate to severe psoriasis. J Am Acad Dermatol. 2015;72:589-98.

27. Sudhapriyadharshini G. Topical glucocorticoids-a review. J Pharm Sci Res. 2014;6:244-6.

28. Bereshchenko O, Bruscoli S, Riccardi C. Glucocorticoids, sex hormones, and immunity. Front Immunol. 2018;9:1332

29. Lahiri KA. Treatise on topical corticosteroids in dermatology. In: Lahiri K, editor. A treatise on topical corticosteroids in dermatology: use, misuse and abuse. Singapore: Springer Singapore; 2018. p. 1-333.

30. Mehta AB, Nadkarni NJ, Patil SP, Godse KV, Gautam M, Agarwal S. Topical corticosteroids in dermatology. Indian $\mathrm{J}$ Dermatol Venereol Leprol. 2016;82:371-8.

31. Coondoo A, Phiske M, Verma S, Lahiri K. Side-effects of topical steroids: a long overdue revisit. Indian Dermatol Online J. 2014;5:416.

32. Dey V. Misuse of topical corticosteroids: a clinical study of adverse effects. Indian Dermatol Online J. 2014;5:436.

33. Rapalli VK, Singhvi G, Dubey SK, Gupta G, Chellappan DK, Dua K. Emerging landscape in psoriasis management: from topical application to targeting biomolecules. Biomed Pharmacother. 2018;106:707-13.

34. Jeevanandam J, Chan YS, Danquah MK. Nano-formulations of drugs: recent developments, impact and challenges. Biochimie. 2016;128-129:99-112.

35. Rai VK, Mishra N, Yadav KS, Yadav NP. Nanoemulsion as pharmaceutical carrier for dermal and transdermal drug delivery: Formulation development, stability issues, basic considerations and applications. J Control Release. 2018;270:203-25.

36. Jijie R, Barras A, Boukherroub R, Szunerits S. Nanomaterials for transdermal drug delivery: beyond the state of the art of liposomal structures. J Mater Chem B. 2017;5:8653-75.

37. Arora S, Lamba H, Tiwari R. Dermal delivery of drugs using different vesicular carriers: a comparative review. Asian J Pharm. 2012;6:237-44.

38. Pierre MBR, Dos Santos Miranda Costa I. Liposomal systems as drug delivery vehicles for dermal and transdermal applications. Arch Dermatological Res Arch Dermatol Res. 2011;303:607-21.

39. Jain S, Jain V, Mahajan SC. Lipid based vesicular drug delivery systems. Adv Pharm. 2014;2014:1-12.

40. Mezei M, Gulasekharam V. Liposomes-a selective drug delivery system for the topical route of administration: gel dosage form. J Pharm Pharmacol. 1982;34:473-4.

41. Mezei M, Gulasekharam V. Liposomes-a selective drug delivery system for the topical route of administration. Lotion Dos form Life Sci. 1980;26:1473-7.

42. Clares B, Gallardo V, Medina MM, Ruiz MA. Multilamellar liposomes of triamcinolone acetonide: Preparation, stability, and characterization. J Liposome Res. 2009;19:197-206.

43. Moldovon M, Leucuta SE, Bakri A. Preparation, in vitro release and skin absorption of hydrocortisone acetate from liposomal gel. J Drug Deliv Sci Technol. 2016;16:127-32.

44. Eroğlu İ, Azizoğlu E, Özyazıcı M, Nenni M, Gürer Orhan H, Özbal S, et al. Effective topical delivery systems for corticosteroids: dermatological and histological evaluations. Drug Deliv. 2016;23:1502-13.

45. Khan I, Yousaf S, Subramanian S, Korale O, Alhnan MA, Ahmed W, et al. Proliposome powders prepared using a slurry method for the generation of beclometasone dipropionate liposomes. Int J Pharm. 2015;496:342-50.
46. Zhang Y, Li H, Du N, Song S, Hou W. Betamethasone dipropionate intercalated layered double hydroxide and the composite with liposome for improved water dispersity. Appl Clay Sci. 2017;143:336-44.

47. Murthy RSR, Rao G. Evaluation of liposomal clobetasol propionate topical formulation for intra-dermal delivery. Indian $\mathrm{J}$ Pharm Sci. 2000;62:459-62.

48. Shah P, Goodyear B, Haq A, Puri V, Michniak-Kohn B. Evaluations of quality by design $(\mathrm{QbD})$ elements impact for developing niosomes as a promising topical drug delivery platform. Pharmaceutics. 2020;12:246

49. Lingan MA, Sathali AA, Kumar MRV, Gokila A. Formulation and evaluation of topical drug delivery system containing clobetasol propionate niosomes. Sci Rev Chem Commun. 2011;1:7-12.

50. Sankar V, Babu E, Siram K, Penmetsa S, Kabila B, Srinavas C, et al. Formulation and clinical evaluation of triamcinolone acetonide niosomes: effect of iontophoresis on the permeation across skin. Pharm Nanotechnol. 2013;1:282-9.

51. Sankar V, Praveen C, Prasanth KG, Srinivas CR, Ruckmann K. Formulation and evaluation of a proniosome hydrocortisone gel in comparison with a commercial cream. Pharmazie. 2009;64:731-4.

52. Vishkaei MN, Ahamed MBK, Majid AMSA. Development of ethosome formulation for topical therapeutic applications. In: Novel drug delivery systems for phytoconstituents. Boca Raton: CRC Press; 2019. p. 137-56.

53. Korade S, Deshmukh MT, Shete RV. Formulation and evaluation of ethosomal gel containing clobetasol. Eur J Pharm Med Res. 2016;2:54-9.

54. Akhtar N, Verma A, Pathak K. Feasibility of binary composition in development of nanoethosomal glycolic vesicles of triamcinolone acetonide using Box-Behnken design: in vitro and ex vivo characterization. Artif Cells, Nanomed Biotechnol. 2017;45:1123-31.

55. Fernández-García R, Lalatsa A, Statts L, Bolás-Fernández F, Ballesteros MP, Serrano DR. Transferosomes as nanocarriers for drugs across the skin: quality by design from lab to industrial scale. Int J Pharm. 2020;573:118817.

56. El Maghraby GM, Barry BW, Williams AC. Liposomes and skin: from drug delivery to model membranes. Eur J Pharm Sci. 2008;34:203-22.

57. Cevc G, Blume G. Hydrocortisone and dexamethasone in very deformable drug carriers have increased biological potency, prolonged effect, and reduced therapeutic dosage. Biochim Biophys Acta - Biomembr. 2004;1663:61-73.

58. Cevc G, Blume G. Biological activity and characteristics of triamcinolone-acetonide formulated with the self-regulating drug carriers, Transfersomes . Biochim Biophys Acta - Biomembr. 2003;1614:156-64.

59. Gillet A, Grammenos A, Compère P, Evrard B, Piel G. Development of a new topical system: drug-in-cyclodextrin-indeformable liposome. Int J Pharm. 2009;380:174-80.

60. Kumar ST, Kurnool AN, Goudanavar P, Acharya A, Sriniwas, Vinay $\mathrm{CH}$, et al. Formulation and evaluation of dexamethasone loaded transfersomal gel by modified hand shaking. World J Pharm Pharm Sci. 2019;8:1181-94.

61. Patel DM, Jani RH, Patel CN. Ufasomes: a vesicular drug delivery. Syst Rev Pharm. 2011;2:72-8.

62. Mittal R, Sharma A, Arora S. Ufasomes mediated cutaneous delivery of dexamethasone: formulation and evaluation of antiinflammatory activity by Carrageenin-induced Rat Paw Edema Model. J Pharm. 2013;2013:1-12.

63. Kumar R. Lipid-based nanoparticles for drug-delivery systems. In: Nanocarriers for drug delivery. Elsevier Inc.; 2019. p. 249-284. 
64. Pradhan M, Singh D, Singh MR. Influence of selected variables on fabrication of Triamcinolone acetonide loaded solid lipid nanoparticles for topical treatment of dermal disorders. Artif Cells, Nanomed Biotechnol. 2016;44:392-400.

65. Pradhan M, Singh D, Singh MR. Development characterization and skin permeating potential of lipid based novel delivery system for topical treatment of psoriasis. Chem Phys Lipids. 2015;186:9-16.

66. Bikkad ML, Nathani AH, Mandlik SK, Shrotriya SN, Ranpise NS. Halobetasol propionate-loaded solid lipid nanoparticles (SLN) for skin targeting by topical delivery. J Liposome Res. 2014;24:113-23.

67. Madan J, Dua K, Khude P. Development and evaluation of solid lipid nanoparticles of mometasone furoate for topical delivery. Int J Pharm Investig. 2014;4:60.

68. Zhang J, Smith E. Percutaneous permeation of betamethasone 17 -valerate incorporated in lipid nanoparticles. J Pharm Sci. 2011;100:896-903.

69. Kalariya M, Padhi BK, Chougule M, Misra A. Clobetasol propionate solid lipid nanoparticles cream for effective treatment of eczema: formulation and clinical implications. Indian J Exp Biol. 2005;43:233-40.

70. Schlupp P, Blaschke T, Kramer KD, Höltje HD, Mehnert W, Schäfer-Korting M. Drug release and skin penetration from solid lipid nanoparticles and a base cream: a systematic approach from a comparison of three glucocorticoids. Ski Pharm Physiol. 2011;24:199-209.

71. Cavalli R, Peira E, Caputo O, Gasco MR. Solid lipid nanoparticles as carriers of hydrocortisone and progesterone complexes with $\beta$-cyclodextrins. Int J Pharm. 1999;182:59-69.

72. Attama AA, Momoh MA, Builders PF. Lipid nanoparticulate drug delivery systems: a revolution in dosage form design and development. In: Recent advances in novel drug carrier systems. London: IntechOpen; 2012.

73. Pradhan M, Alexander A, Singh MR, Singh D, Saraf S, Saraf S, et al. Understanding the prospective of nano-formulations towards the treatment of psoriasis. Biomed Pharmacother. 2018;107:447-63.

74. Pradhan M, Singh D, Murthy SN, Singh MR. Corrigendum to "Design, characterization and skin permeating potential of Fluocinolone acetonide loaded nanostructured lipid carriers for topical treatment of psoriasis" [Steroids 101 (2015) 56-63] (S0039128X15001762). Steroids. 2016;106:93 https://doi.org/ 10.1016/j.steroids.2015.05.012

75. Pradhan M, Singh D, Singh MR. Fabrication, optimization and characterization of Triamcinolone acetonide loaded nanostructured lipid carriers for topical treatment of psoriasis: application of Box Behnken design, in vitro and ex vivo studies. $\mathbf{J}$ Drug Deliv Sci Technol. 2017;41:325-33.

76. Carvajal-Vidal P, Fábrega MJ, Espina M, Calpena AC, García ML. Development of Halobetasol-loaded nanostructured lipid carrier for dermal administration: optimization, physicochemical and biopharmaceutical behavior, and therapeutic efficacy. Nanomed Nanotechnol Biol Med. 2019;20:102026.

77. Silva LAD, Andrade LM, De Sá FAP, Marreto RN, Lima EM, Gratieri T, et al. Clobetasol-loaded nanostructured lipid carriers for epidermal targeting. J Pharm Pharm. 2016;68:742-50.

78. Escobar-Chávez JJ. Current technologies to increase the transdermal delivery of drugs. Mexico: Bentham Science Publishers Ltd.; 2010.

79. Nam YS, Kim JW, Park JY, Shim J, Lee JS, Han SH. Tocopheryl acetate nanoemulsions stabilized with lipid-polymer hybrid emulsifiers for effective skin delivery. Colloids Surf B Biointerfaces. 2012;94:51-7.

80. Mason TG, Wilking JN, Meleson K, Chang CB, Graves SM. Nanoemulsions: formation, structure, and physical properties. J Phys Condens Matter. 2006;18:R635-66.
81. Komaiko JS, Mcclements DJ. Formation of food-grade nanoemulsions using low-energy preparation methods: a review of available methods. Compr Rev Food Sci Food Saf. 2016;15:331-52.

82. Baspinar Y, Keck CM, Borchert HH. Development of a positively charged prednicarbate nanoemulsion. Int $\mathrm{J}$ Pharm. 2010;383:201-8.

83. Alam MS, Ali MS, Alam N, Alam MI, Anwer T, Imam F, et al. Design and characterization of nanostructure topical gel of betamethasone dipropionate for psoriasis. J Appl Pharm Sci. 2012;2:148-58.

84. Sajid Ali M, Sarfaraz Alam M, Alam N, Akmal Shah Qadry S, Intakhab Alam M, Shamim M, et al. Formulation, characterization and in-vivo assessment of topical nanoemulsion of betamethasone valerate for psoriasis and dermatose. Int $\mathrm{J}$ Pharm. 2013;3:186-99.

85. Alam MS, Ali MS, Zakir F, Alam N, Alam MI, Ahmad F, et al. Enhancement of anti-dermatitis potential of clobetasol propionate by DHA [docosahexaenoic acid] rich algal oil nanoemulsion gel. Iran J Pharm Res. 2016;15:35-52.

86. Melero A, Ferreira Ourique A, Stanisçuaski Guterres S, Raffin Pohlmann A, Lehr CM, Ruver Beck RC, et al. Nanoencapsulation in lipid-core nanocapsules controls mometasone furoate skin permeability rate and its penetration to the deeper skin layers. Ski Pharm Physiol. 2014;27:217-28.

87. Teixeira Z, Zanchetta B, Melo BAG, Oliveira LL, Santana MHA, Paredes-Gamero EJ, et al. Retinyl palmitate flexible polymeric nanocapsules: characterization and permeation studies. Colloids Surf B Biointerfaces. 2010;81:374-80.

88. Erdoğar N, Akkın S, Bilensoy E. Nanocapsules for drug delivery: an updated review of the last decade. Recent Pat Drug Deliv Formul. 2019;12:252-66.

89. Fontana MC, Rezer JFP, Coradini K, Leal DBR, Beck RCR. Improved efficacy in the treatment of contact dermatitis in rats by a dermatological nanomedicine containing clobetasol propionate. Eur J Pharm Biopharm. 2011;79:241-9.

90. Beber TC, De Andrade DF, Dos Santos Chaves P, Pohlmann AR, Guterres SS, Ruver, et al. Cationic polymeric nanocapsules as a strategy to target dexamethasone to viable epidermis: skin penetration and permeation studies. J Nanosci Nanotechnol. 2016;16:1331-8.

91. Antonow MB, Lorenzoni R, Barbosa GM, Ourique AF, Gomes P, Raffin RP. Development and physicochemical characterization of desonide-loaded nanocapsule suspensions. Adv Mater Sci Eng. 2016;7395896.

92. Assem M, Khowessah OM, Ghorab D. Optimization and evaluation of beclomethasone dipropionate micelles incorporated into biocompatible hydrogel using a sub-chronic dermatitis animal model. AAPS PharmSciTech. 2019;20:1-12.

93. Letchford $\mathrm{K}$, Burt $\mathrm{H}$. A review of the formation and classification of amphiphilic block copolymer nanoparticulate structures: micelles, nanospheres, nanocapsules and polymersomes. Eur J Pharm Biopharm. 2007;65:259-69.

94. Saralidze K, Koole LH, Knetsch MLW. Polymeric microspheres for medical applications. Materials. 2010;3:3537-64.

95. Badıllı U, Şen T, Tarımcı N. Microparticulate based topical delivery system of clobetasol propionate. AAPS PharmSciTech. 2011;12:949-57.

96. Mahant S, Kumar S, Nanda S, Rao R. Microsponges for dermatological applications: perspectives and challenges. Asian J Pharm Sci. 2019;15:273-91.

97. Kaity S, Maiti S, Ghosh AK, Pal D, Ghosh A, Banerjee S. Microsponges: a novel strategy for drug delivery system. J Adv Pharm Technol Res. 2010;1:283-90.

98. Mohanty D, Bakshi V, Rashaid MA, Reddy TV, Nidhi A. Design and in-vitro characterization of betamethasone microsponge loaded topical gel. Int J Pharma Res Heal Sci. 2016;4:1124-9. 
99. Rekha U, Manjula BP. Formulation and evaluation of microsponges for topical drug delivery of mometasone furoate. Int $\mathbf{J}$ Pharm Pharm Sci. 2011;3:133-7.

100. Devi N, Kumar S, Prasad M, Rao R. Eudragit RS100 based microsponges for dermal delivery of clobetasol propionate in psoriasis management. J Drug Deliv Sci Technol. 2020;55:101347.

101. D'souza JI, More HN. Topical anti-inflammatory gels of fluocinolone acetonide entrapped in Eudragit based microsponge delivery system. Res J Pharm Tech. 2008;1:502-6.

102. Jahangiri A, Davaran S, Fayyazi B, Tanhaei A, Payab S, Adibkia $\mathrm{K}$. Application of electrospraying as a one-step method for the fabrication of triamcinolone acetonide-PLGA nanofibers and nanobeads. Colloids Surf B Biointerfaces. 2014;123:219-24.

103. Osada M, Sasaki T. Two-dimensional dielectric nanosheets: novel nanoelectronics from nanocrystal building blocks. Adv Mater. 2012;24:210-28.

104. Mitter N, Worrall EA, Robinson KE, Li P, Jain RG, Taochy C, et al. Clay nanosheets for topical delivery of RNAi for sustained protection against plant viruses. Nat Plants. 2017;3:16207

105. Hatanaka T, Saito T, Fukushima T, Todo H, Sugibayashi K, Umehara $\mathrm{S}$, et al. Potential of biocompatible polymeric ultra-thin films, nanosheets, as topical and transdermal drug delivery devices. Int J Pharm. 2019;565:41-9.

106. Rosado C, Silva C, Reis CP. Hydrocortisone-loaded poly( $\varepsilon$ caprolactone) nanoparticles for atopic dermatitis treatment. Pharm Dev Technol. 2013;18:710-8.

107. Zhang Z, Tsai PC, Ramezanli T, Michniak-Kohn BB. Polymeric nanoparticles-based topical delivery systems for the treatment of dermatological diseases. Wiley Interdiscip Rev: Nanomed Nanobiotechnol. 2013;5:205-18.

108. Felt O, Buri P, Gurny R. Chitosan: a unique polysaccharide for drug delivery. Drug Dev Ind Pharm 1998;24:979-93.

109. Hussain Z, Katas H, Amin MCIM, Kumulosasi E, Sahudin S. Antidermatitic perspective of hydrocortisone as chitosan nanocarriers: an ex vivo and in vivo assessment using an NC/Nga mouse model. J Pharm Sci. 2013;102:1063-75.

110. Özcan I, Azizoğlu E, Senyiğit T, Özyazici M, Özer Ö. Enhanced dermal delivery of diflucortolone valerate using lecithin/chitosan nanoparticles: in-vitro and in-vivo evaluations. Int $\mathbf{J}$ Nanomed. 2013;8:461-75.

111. Özcan I, Azizoğlu E, Şenyiğit T, Özyazici M, Özer Ö. Comparison of PLGA and lecithin/chitosan nanoparticles for dermal targeting of betamethasone valerate. J Drug Target. 2013;21:542-50.

112. Şenyiğit T, Sonvico F, Rossi A, Tekmen I, Santi P, Colombo P, et al. In vivo assessment of clobetasol propionate-loaded lecithinchitosan nanoparticles for skin delivery. Int $\mathrm{J}$ Mol Sci. 2017;18:1-12.

113. Fülöp $Z$, Saokham $P$, Loftsson T. Sulfobutylether$\beta$-cyclodextrin/chitosan nano- and microparticles and their physicochemical characteristics. Int J Pharm. 2014;472:282-7.

114. Ayano E, Karaki M, Ishihara T, Kanazawa H, Okano T. Poly (Nisopropylacrylamide)-PLA and PLA blend nanoparticles for temperature-controllable drug release and intracellular uptake. Colloids Surf B Biointerfaces. 2012;99:67-73.
115. Ishihara T, Izumo N, Higaki M, Shimada E, Hagi T, Mine L, et al. Role of zinc in formulation of PLGA/PLA nanoparticles encapsulating betamethasone phosphate and its release profile. J Control Release. 2005;105:68-76.

116. Ghasemnejad M, Ahmadi E, Mohamadnia Z, Doustgani A, Hashemikia S. Functionalized silica nanoparticles as a carrier for betamethasone sodium phosphate: drug release study and statistical optimization of drug loading by response surface method. Mater Sci Eng C. 2015;56:223-32.

117. Li Y, Chen X, Xia L, Xiao X, Li G. Magnetic metal-organic frameworks-101 functionalized with graphite-like carbon nitride for the efficient enrichment of glucocorticoids in cosmetics. J Chromatogr A. 2019;1606:460382

118. Wang Y, Cheetham AG, Angacian G, Su H, Xie L, Cui H. Peptide-drug conjugates as effective prodrug strategies for targeted delivery. Adv Drug Deliv Rev. 2017;110-111:112-26.

119. Dolz-Pérez I, Sallam MA, Masiá E, Morelló-Bolumar D, Pérez del Caz MD, Graff P, et al. Polypeptide-corticosteroid conjugates as a topical treatment approach to psoriasis. J Control Release. 2020;318:210-22.

120. Baboota S, Sharma S, Kumar A, Alam MS, Sahni J, Ali J. Nanocarrier-based hydrogel of betamethasone dipropionate and salicylic acid for treatment of psoriasis. Int J Pharm Investig. 2011;1:139.

121. Kaur A, Katiyar SS, Kushwah V, Jain S. Nanoemulsion loaded gel for topical co-delivery of clobitasol propionate and calcipotriol in psoriasis. Nanomed Nanotechnol Biol Med. 2017;13:1473-82.

122. Sonawane R, Harde H, Katariya M, Agrawal S, Jain S. Solid lipid nanoparticles-loaded topical gel containing combination drugs: an approach to offset psoriasis. Expert Opin Drug Deliv. 2014;11:1833-47.

123. Siddique MI, Katas H, Amin MCIM, Ng SF, Zulfakar MH, Buang F, et al. Minimization of local and systemic adverse effects of topical glucocorticoids by nanoencapsulation: in vivo safety of hydrocortisone-hydroxytyrosol loaded chitosan nanoparticles. J Pharm Sci. 2015;104:4276-86.

124. Bhat M, Pukale S, Singh S, Mittal A, Chitkara D. Nano-enabled topical delivery of anti-psoriatic small molecules. J Drug Deliv Sci Technol. 2021;62:102328.

125. Rahman M, Akhter S, Ahmad J, Ahmad MZ, Beg S, Ahmad FJ. Nanomedicine-based drug targeting for psoriasis: potentials and emerging trends in nanoscale pharmacotherapy. Expert Opin Drug Deliv. 2015;12:635-52.

126. Senyigit T, Ozer O. Corticosteroids for skin delivery: challenges and new formulation opportunities. In: Glucocorticoids-new recognition of our familiar friend. London: IntechOpen; 2012.

127. Hussain Z, Katas H, Mohd Amin MCI, Kumolosasi E, Buang F, Sahudin S. Self-assembled polymeric nanoparticles for percutaneous co-delivery of hydrocortisone/hydroxytyrosol: an ex vivo and in vivo study using an NC/Nga mouse model. Int J Pharm. 2013;444:109-19.

128. World Health Organization. WHO model prescribing information: drugs used in skin diseases. A World Health Organization resource. 1997. 